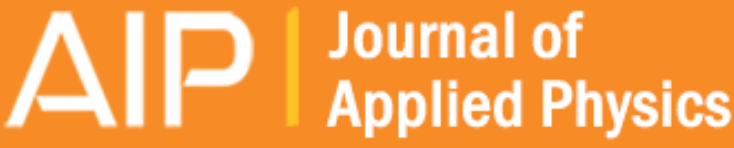

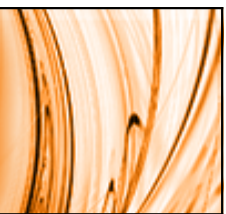

Impurity-limited resistance and phase interference of localized impurities under quasione dimensional nano-structures

Nobuyuki Sano

Citation: Journal of Applied Physics 118, 244302 (2015); doi: 10.1063/1.4938392

View online: http://dx.doi.org/10.1063/1.4938392

View Table of Contents: http://scitation.aip.org/content/aip/journal/jap/118/24?ver=pdfcov

Published by the AIP Publishing

\section{Articles you may be interested in}

Theoretical impurity-limited carrier mobility of monolayer black phosphorus

Appl. Phys. Lett. 108, 033508 (2016); 10.1063/1.4940381

Decoupling single nanowire mobilities limited by surface scattering and bulk impurity scattering

J. Appl. Phys. 110, 033705 (2011); 10.1063/1.3611032

Lattice thermal conductivity of freestanding gallium nitride nanowires

J. Appl. Phys. 108, 034324 (2010); 10.1063/1.3463358

Effects of impurity scattering on the quantized conductance of a quasi-one-dimensional quantum wire Appl. Phys. Lett. 94, 012105 (2009); 10.1063/1.3067995

Impurity-limited mobility of semiconducting thin wires in $\mathrm{n}$-type gallium arsenide J. Appl. Phys. 83, 1390 (1998); 10.1063/1.366842

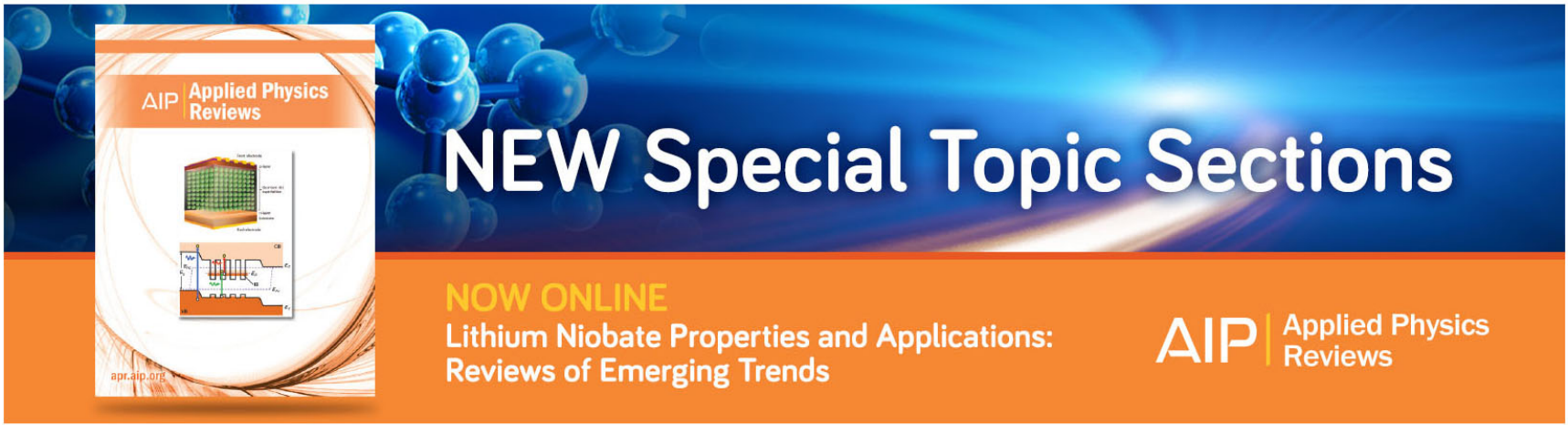




\title{
Impurity-limited resistance and phase interference of localized impurities under quasi-one dimensional nano-structures
}

\author{
Nobuyuki Sano ${ }^{a)}$ \\ Institute of Applied Physics, University of Tsukuba, 1-1-1 Tennodai, Tsukuba, Ibaraki 305-8573, Japan
}

(Received 27 October 2015; accepted 9 December 2015; published online 24 December 2015)

\begin{abstract}
The impurity-limited resistance and the effect of the phase interference among localized multiple impurities in the quasi-one dimensional (quasi-1D) nanowire structures are systematically investigated under the framework of the scattering theory. We derive theoretical expressions of the impurity-limited resistance in the nanowire under the linear response regime from the Landauer formula and from the Boltzmann transport equation (BTE) with the relaxation time approximation. We show that the formula from the BTE exactly coincides with that from the Landauer approach with the weak-scattering limit when the energy spectrum of the in-coming electrons from the reservoirs is narrow and, thus, point out a possibility that the distinction of the impurity-limited resistances derived from the Landauer formula and that of the BTE could be made clear. The derived formulas are applied to the quasi-1D nanowires doped with multiple localized impurities with short-range scattering potential and the validity of various approximations on the resistance are discussed. It is shown that impurity scattering becomes so strong under the nanowire structures that the weak-scattering limit breaks down in most cases. Thus, both phase interference and phase randomization simultaneously play a crucial role in determining the impurity-limited resistance even under the fully coherent framework. When the impurity separation along the wire axis direction is small, the constructive phase interference dominates and the resistance is much greater than the average resistance. As the separation becomes larger, however, it approaches the series resistance of the single-impurity resistance due to the phase randomization. Furthermore, under the uniform configuration of impurities, the space-average resistance of multiple impurities at room temperature is very close to the series resistance of the single-impurity resistance, and thus, each impurity could be regarded as an independent scattering center. The physical origin of this "self-averaging" under the fully coherent environments is attributed to the broadness of the energy spectrum of the in-coming electrons from the reservoirs. (C) 2015 AIP Publishing LLC.
\end{abstract}

[http://dx.doi.org/10.1063/1.4938392]

\section{INTRODUCTION}

Silicon nanowires (SNWs) have been receiving great attention because of their possible application of future electronic and photonic devices as well as other novel applications such as chemical/biological sensors. ${ }^{1-3}$ In particular, SNWs with gate-all-around structure could be fabricated with diameter less than $10 \mathrm{~nm}$ and show excellent transistor characteristics. ${ }^{4-6}$ Therefore, SNWs are considered to be one of the most promising building blocks for future nanotransistors because of their excellent gate-controllability. ${ }^{7,8}$

Transport characteristics of nanowires observed in various experiments are, however, scattered over great ranges. One of the main causes is due to the small volume through which electrons flow so that the number of impurities and surface roughness included in the volume is very small. ${ }^{9-11}$ Since "self-averaging," by which the resistance is averaged over the random spatial configuration of impurities and/or surface roughness, ${ }^{12,13}$ is no longer valid in short channel nanowires, the device performance greatly fluctuates depending on the spatial configuration of surface roughness and impurities in the channel. ${ }^{14,15}$ In fact, this is a rather

${ }^{a)}$ Electronic mail: sano@esys.tsukuba.ac.jp complicated problem because both incoherent and coherent effects associated with electron's wave nature simultaneously play a role in the variability of device properties: the quantum phase interference along the longitudinal direction as well as the potential modulation associated with localized impurities and surface roughness is deeply involved. In addition, because of the singular nature inherent in the transport equation, scattering is inevitable even in nano-scale channel devices, in which the mean-free-path is much longer than the channel length. ${ }^{16-18}$ So far, most theoretical studies on electron transport properties of SNWs are based on large-scale numerical simulations, as briefly overviewed below, and the effects of phase interference among ionized impurities on electron transport and their consequence of "self-averaging" are not fully explored or understood.

The effects of localized impurities on transport characteristics under nanowire structures have been tackled by quantum mechanical simulations such as the nonequilibrium Green function (NEGF). The electronic structure of localized impurities is obtained from either the first-principles calculations with the density-functional theory (DFT) ${ }^{19-23}$ or the empirical tight-binding method. ${ }^{24-26}$ In both approaches, the conductance is calculated by the Landauer formula in terms of the transmission probability under the framework of the 
scattering theory. Under the low dimensional structures, the electronic structure becomes rather sensitive to the atomistic constituents of the device and the electrostatic potential is greatly modulated by the difference in dielectric properties of the surrounding materials (dielectric mismatch). In particular, this dielectric mismatch affects the screening of the Coulomb scattering potential of ionized impurities, leading to a strong dependence of the electron mobility on the surrounding materials. ${ }^{27-29}$

Although charged impurities sometimes lead to numerical convergence problems in the first-principles calculations, ${ }^{21}$ these approaches indeed provide accurate electronic structures and potential profiles under nanostructure once proper boundary conditions are imposed on device geometry. However, the incorporation of the whole device structure including the surroundings is very difficult, and the number of impurities included in such calculations is rather limited due to the current computational capability. In many cases, just one single-impurity is put at various location in the transverse plane of the quasi-one dimensional (quasi-1D) nanowire and the impurity-limited resistance (or equivalently the mobility) is extracted. The mobility is found to be strongly dependent of the type of charge and the radial position of the ionized impurity in the nanowire. ${ }^{24,26}$ It is, thus, claimed that the perturbative treatment of impurity scattering based on the Boltzmann transport equation (BTE) would completely fail to predict the accurate mobilities. It should be noted, however, that the analyses based on a single impurity could be justified only if each impurity doped in the nanowire is regarded as independent so that the phase correlation among the impurities is completely ignored. In addition, self-averaging is implicitly involved in the long channel nanowires usually employed in the experiments and it is not clear whether the self-averaged resistance would be identical to that simply extracted from the single-impurity resistance.

There are several theoretical reports in which multiple impurities, consistently with the average impurity density imposed in the substrate of the nanowire, are introduced explicitly to evaluate the transport properties in the tightbinding NEGF simulations. ${ }^{25,30,31}$ They have demonstrated how much the device characteristics could actually fluctuate, depending on the configuration of impurities. However, the number of simulations capable by the tight-binding approaches is still limited, and it is very difficult to obtain statistically reliable results. In addition, under the framework of the NEGF simulations, the potential modulation induced by multiple impurities over the whole channel region is treated as a "single" scattering potential, and thus, the phase coherence among the impurities is completely preserved unless the phase breaking process such as phonon scattering is explicitly included. Therefore, it is not clear how selfaveraging is actually involved in determining the transport properties. Even if phase breaking scattering is included, it is very difficult to extract the distinct effects of phase interference among impurities from such brute-force simulations. As a result, the physics behind the variability associated with phase interference in transport characteristics is not clear and its full understanding is still missing.
In the present paper, we carry out systematic investigations of the impurity-limited resistance and the effect of the phase interference among localized multiple impurities in the quasi-1D nanowire structures.

We derive theoretical expressions of the impuritylimited resistance in the nanowire under the linear response regime from the Landauer formula and the BTE with the relaxation time approximation. We show that the formula from the BTE exactly coincides with that from the Landauer approach with the weak-scattering limit under certain conditions. We point out a possibility that the distinction of the impurity-limited resistances derived from the Landauer formula and that of the BTE could be made clear experimentally. The derived formulas are then applied to the quasi-1D nanowires doped with multiple localized impurities with short-range scattering potential. The range of validity of various approximations on the impurity-limited resistance is discussed. In order to clarify the physics behind the variability associated with localized impurities in the nanowire, numerical analyses are carried out based on these formulas, and we show explicitly that both phase interference and phase randomization play a crucial role simultaneously in determining the impurity-limited resistance even under the fully coherent framework where no phase breaking scattering is included. We also show that under the uniform distribution of impurities, the space-average resistance at room temperature becomes very close to the series resistance of the singleimpurity resistance, and thus, each impurity could be regarded as an independent scattering center. We discuss the physical origin of self-averaging under purely coherent circumstances.

The present paper is organized as follows. The details of the theoretical methodology are explained in Sec. II: The impurity-limited resistances are derived from the Landauer formula and the BTE. The reflection and transmission probabilities are derived from the Lippmann-Schwinger theory. The exact expressions of the resistance in the nanowire due to multiple impurities with short-range potential are explicitly derived. In Sec. III, the derived formulas are applied to the simple cases where single and multiple impurities are doped in the nanowire, and the range of validity of various approximations and the phase interference effects are discussed. Finally, some conclusions from the present study are drawn in Sec. IV.

\section{THEORETICAL METHODOLOGY}

\section{A. Landauer formula and impurity-limited resistance}

We consider a quasi-1D quantum wire which has the spherical cross-section with radius $r_{s}$. The unperturbed Hamiltonian $\hat{H}_{0}$ under the effective mass approximation for the cylindrical wire is expressed as

$$
\hat{H}_{0}=-\frac{\hbar^{2}}{2 m} \nabla^{2}+U_{c y l}(\hat{\mathbf{R}})
$$

where $m$ is the electron effective mass and $U_{c y l}(\hat{\mathbf{R}})$ is the single-particle potential energy, which confines electrons inside the cylindrical wire. In fact, $\mathrm{Si}$ has 6 equivalent 
valleys with anisotropic effective masses in bulk, which lead to the subband splitting under nanostructures. In principle, the extension to anisotropic band structure is also possible by introducing the anisotropic effective masses in the above Hamiltonian. However, since the emphasis in the present study is paid on the physics involved in localized impurities in conventional semiconductors, we employ an isotropic band structure with single-valley for simplicity. The wavefunction in the cylindrical coordinates is then given by

$$
\begin{aligned}
\phi_{k l n}(\mathbf{R}) & =\frac{1}{\sqrt{L}} e^{i k z} \xi_{\ln }(\mathbf{r}) \\
& =\frac{1}{\sqrt{L}} e^{i k z} \frac{1}{\sqrt{\pi} r_{s} J_{l+1}\left(x_{l n}\right)} J_{l}\left(x_{\ln } \frac{r}{r_{s}}\right) e^{i l \varphi},
\end{aligned}
$$

where $\mathbf{R}=(\mathbf{r}, z)=(r, \varphi, z), L$ is the wire (channel) length, $J_{l}(x)$ is the Bessel function of order $l$ (integer), and $x_{l n}$ is the $n$-th root of $J_{l}(x)=0$. The total electron energy is given by

$$
E_{k l n}=\varepsilon_{k}+\varepsilon_{l n}=\frac{\hbar^{2} k^{2}}{2 m}+\frac{\hbar^{2}}{2 m}\left(\frac{x_{l n}}{r_{s}}\right)^{2} .
$$

According to the Landauer picture, the electrical current $I$ through the nanowire connected to the two reservoirs with chemical potential $\mu_{L}$ for the left (source) and $\mu_{R}$ for the right (drain) is expressed by

$$
I=\frac{e}{\pi \hbar} \int_{-\infty}^{\infty} d E \sum_{A} T_{A}(E)\left\{f_{F D}\left(E ; \mu_{L}\right)-f_{F D}\left(E ; \mu_{R}\right)\right\},
$$

where $T_{A}(E)$ is the transmission coefficient (probability) for the incoming electron in the subband $A=(l, n)$ with total energy $E(>0)$ and $f_{F D}(E ; \mu)$ is the Fermi-Dirac distribution with the chemical potential $\mu$. The spin degeneracy is included in the above expression. Under the linear response regime where the applied bias $V$ is small, we obtain the Landauer formula for the conductance $G$ as

$$
G=\frac{e^{2}}{\pi \hbar} \int_{-\infty}^{\infty} d E \sum_{A} T_{A}(E)\left(-\frac{\partial f_{F D}(E)}{\partial E}\right),
$$

where we have eliminated the chemical potential $\mu_{L}$ from its argument of the Fermi-Dirac distribution $f_{F D}$ for simplicity.

The total resistance $R_{\text {tot }}$ given by the inverse of $G$ in Eq. (5) consists of two contributions: the contact (quantum) resistance $R_{0}$ and the channel resistance $R_{c}$. The former is caused by the difference in the number of modes in the reservoirs and the lead. On the other hand, $R_{c}$ is associated with the scattering potential by ionized impurities, phonons, surface roughness, and the potential modulation, resulting from the long range part of the Coulomb potential of ionized impurities and carriers as well as the applied gate voltage. ${ }^{32}$ Therefore, the channel resistance $R_{c}$ could be obtained by subtracting the contact resistance from the total resistance

$$
R_{c}=R_{t o t}-R_{0}=\frac{1}{G}-\frac{1}{G_{0}} .
$$

The contact resistance $R_{0}$ would be given by simply assuming that the transmission probability $T_{A}(E)$ is unity (ballistic) and, thus, the channel resistance $R_{c}$ is calculated by

$$
R_{C}=\frac{\pi \hbar}{e^{2}} \frac{1}{g_{s u b}} \frac{\left\langle\sum_{A} R_{A}(E)\right\rangle}{\left\langle\sum_{A} T_{A}(E)\right\rangle},
$$

where $g_{s u b}$ is the number of subbands (modes) available for the in-coming electrons from the source and the drain with the Fermi energy $\mu_{L}$ and $R_{A}(E)$ is the reflection coefficient (probability) for the electron with energy $E$. We have used the fact that $T_{A}(E)+R_{A}(E)=1 .\langle\cdots\rangle$ represents the thermal average defined by

$$
\langle\cdots\rangle=\int_{-\infty}^{\infty} d E(\cdots)\left(-\frac{\partial f_{F D}(E)}{\partial E}\right) .
$$

If the Fermi energy is close to or below the conduction band edge in the channel region, Eq. (8) is not normalized to unity. Thus, the averaged quantities such as $\left\langle R_{A}\right\rangle$ should be interpreted as those properly normalized.

It should be noted that the scattering-limited resistance $R_{c}$ in Eq. (7) has no lower or upper bound, as it should be, and approaches zero (or infinity) as $R_{A}(E)$ (or $T_{A}(E)$ ) approaches zero. Therefore, Eq. (7) allows us to directly compare the scattering-limited resistance with that calculated from the BTE, which does not include the contact (quantum) resistance and takes the value in the ranges of $[0, \infty)$.

When only the lowest subband, $A=(l=0, n=1)$, is involved in the transport (we call it the "extreme quantum limit" hereafter), $R_{c}$ reduces to the following expression:

$$
\begin{aligned}
R_{c} & =\frac{\pi \hbar}{e^{2}} \frac{\left\langle R_{A}\right\rangle}{1-\left\langle R_{A}\right\rangle}=\frac{\pi \hbar}{e^{2}}\left\{\left\langle R_{A}\right\rangle+\left\langle R_{A}\right\rangle^{2}+\cdots\right\} \\
& \approx \frac{\pi \hbar}{e^{2}}\left\langle R_{A}\right\rangle .
\end{aligned}
$$

The last expression holds true under "the weak-scattering limit" where the transmission probability $\left\langle T_{A}\right\rangle$ is very close to unity, i.e., $\left\langle R_{A}\right\rangle \ll 1$. Therefore, this expression of $R_{c}$ is bounded above and physically inappropriate. We shall show below that the semi-classical treatment based on the BTE under the linear response regime yields the identical results to the Landauer's under the weak-scattering limit.

We should notice that under the framework of the scattering theory, the potential modulation in the whole channel region is regarded as a "single" scattering potential. This potential modulation is attributed to the following two parts. One is the short-range part of the Coulomb potential due to ionized impurities and carriers. In addition, the smooth potential induced by the long-range part of the Coulomb potential of impurities/carriers and the applied gate voltage also contributes to the channel resistance $R_{c}$. Therefore, the channel resistance $R_{c}$ results from the two different origins in the Landauer approach. Figure 1 shows a schematic drawing of a typical potential profile encountered in the channel of nanowire FETs. We would like to stress that under the semiclassical analyses based on the BTE, this long-range potential modulation is not treated as the scattering potential and does not directly contribute to the resistance. It simply affects the 


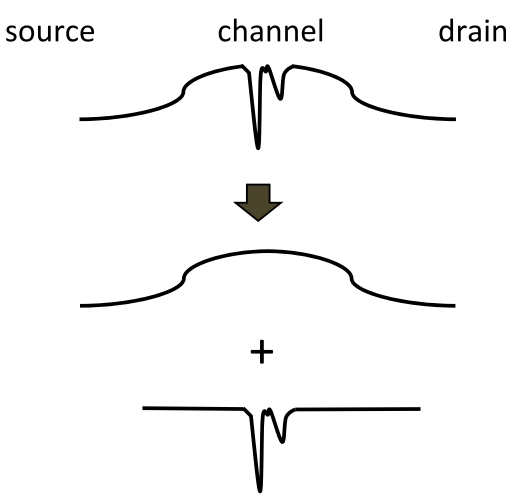

FIG. 1. Schematic drawing of a typical potential profile encountered in the channel of nanowire FETs. The whole scattering potential consists of the long-range potential induced mainly by the applied gate voltage and the short-range potential due to the screened impurities. The entire potential profile is treated as a single-scattering potential under the framework of the scattering theory.

carrier density in the channel region. Therefore, it is crucial to eliminate the long-range part of the scattering potential from the potential modulation in the Landauer approach in order to make the direct comparison with the semi-classical analyses based on the BTE meaningful. In the present study, we consider the transport properties associated with localized impurities with short-range scattering potential under the flat channel potential. If the long-range potential along the wire axis in the channel is flat by properly applying the gate voltage, the channel resistance $R_{c}$ could be identified as the impurity-limited resistance $R_{S}$ by ionized impurities with the short-range screened Coulomb potential. ${ }^{33}$

\section{B. Transmission and reflection probabilities}

The transmission and reflection probabilities, $T_{A}(E)$ and $R_{A}(E)$, could be calculated by the Lippmann-Schwinger equation. ${ }^{34}$ The complete state vector $\left|\psi^{+}(E)\right\rangle$ of the incoming electron with total energy $E$ from the reservoirs under the influence of the full Hamiltonian is described by

$$
\left|\psi^{+}(E)\right\rangle=|\phi(E)\rangle+\hat{G}_{0}^{+}(E) \hat{T}(E)|\phi(E)\rangle
$$

where $|\phi(E)\rangle$ is the unperturbed state vector with energy $E$, which coincides adiabatically with $\left|\psi^{+}(E)\right\rangle$ at very far distant (in space and/or time) from the interaction (channel) region. ${ }^{35,36} \hat{G}_{0}^{+}(E)$ is the free retarded Green operator defined with the unperturbed Hamiltonian $\hat{H}_{0}$, and $\hat{T}(E)$ is the $T$-operator defined by

$$
\hat{T}(E)=\hat{V}+\hat{V} \hat{G}_{0}^{+}(E) \hat{T}(E)
$$

with the interaction operator $\hat{V}$ associated with the scattering potential, which may include the hopping term to connect the unperturbed parts of the whole system.

Projecting Eq. (10) onto the coordinate space, we obtain the complete wave-function as

$$
\begin{aligned}
\psi^{+}(\mathbf{R})= & \frac{1}{\sqrt{L}} e^{i k_{A} z} \xi_{A}(\mathbf{r})+\frac{1}{\sqrt{L}} \sum_{B} \xi_{B}(\mathbf{r}) \\
& \times \int d z_{1} d z_{2} g_{k_{B}}^{+}\left(z, z_{1}\right) \bar{T}_{B A}\left(z_{1}, z_{2}\right) e^{i k_{A} z_{2}}
\end{aligned}
$$

where the eigenstate of the unperturbed Hamiltonian $\hat{H}_{0}$ is denoted by $(E, l, n) \equiv(E, B)$ and $k_{B}=\sqrt{2 m\left(E-\varepsilon_{B}\right)} / \hbar$ $(>0)$. We assume that the electron in the eigenstate $(E, A)$ is injected into the scattering region. The reduced Green function $g_{k_{B}}^{+}\left(z_{1}, z_{2}\right)$ is expressed by

$$
g_{k_{B}}^{+}\left(z_{1}, z_{2}\right)=-i \frac{m}{\hbar^{2} k_{B}} e^{i k_{B}\left|z_{1}-z_{2}\right|},
$$

and the reduced $T$-matrix is given by

$$
\begin{aligned}
\bar{T}_{B A}\left(z_{1}, z_{2}\right)= & \bar{V}_{B A}\left(z_{1}\right) \delta\left(z_{1}-z_{2}\right)+\sum_{C} \bar{V}_{B C}\left(z_{1}\right) \\
& \times g_{k_{C}}^{+}\left(z_{1}, z_{2}\right) \bar{V}_{C A}\left(z_{2}\right)+\sum_{C, D} \bar{V}_{B C}\left(z_{1}\right) \int d z_{3} g_{k_{C}}^{+} \\
& \times\left(z_{1}, z_{3}\right) \bar{V}_{C D}\left(z_{3}\right) g_{k_{D}}^{+}\left(z_{3}, z_{2}\right) \bar{V}_{D A}\left(z_{2}\right)+\cdots
\end{aligned}
$$

Here, the effective interaction potential $\bar{V}_{B A}$ is defined by

$$
\bar{V}_{B A}\left(z_{1}\right)=\int d^{2} r_{1} \xi_{B}^{*}\left(\mathbf{r}_{1}\right) V\left(\mathbf{R}_{1}\right) \xi_{A}\left(\mathbf{r}_{1}\right) .
$$

The asymptotes at $z \rightarrow \pm \infty$ of the complete wavefunction allows one to calculate the transmission and reflection amplitudes, $t_{B A}(E)$ and $r_{B A}(E)$, respectively. The results are given by

$$
t_{B A}(E)=\delta_{B, A}+I_{B A}^{-}
$$

and

$$
r_{B A}(E)=I_{B A}^{+},
$$

where $I_{B A}^{\mp}$ is defined by

$$
I_{B A}^{\mp}=\int d z_{1} d z_{2} e^{\mp i k_{B} z_{1}}\left(-i \frac{m}{\hbar^{2} k_{B}}\right) \bar{T}_{B A}\left(z_{1}, z_{2}\right) e^{i k_{A} z_{2}} .
$$

The transmission and reflection probabilities for the incoming electron with energy $E$ are given by

$$
T_{A}(E)=\sum_{B}\left|t_{B A}(E)\right|^{2} \frac{k_{B}}{k_{A}}
$$

and

$$
R_{A}(E)=\sum_{B}\left|r_{B A}(E)\right|^{2} \frac{k_{B}}{k_{A}},
$$

respectively. The exact impurity-limited resistance $R_{s}$ is evaluated with Eq. (7) and we have

$$
R_{S}=\frac{\pi \hbar}{e^{2}} \frac{1}{g_{\text {sub }}} \frac{\left\langle\sum_{A, B}\left|r_{B A}(E)\right|^{2} \frac{k_{B}}{k_{A}}\right\rangle}{\left\langle\sum_{A, B}\left|t_{B A}(E)\right|^{2} \frac{k_{B}}{k_{A}}\right\rangle} .
$$

In particular, under the extreme quantum limit where only the lowest subband $A$ is involved in electron transport, the exact impurity-limited resistance $R_{S}$ becomes 


$$
R_{S}=\frac{\pi \hbar}{e^{2}} \frac{\left\langle R_{A}(E)\right\rangle}{\left\langle T_{A}(E)\right\rangle}=\frac{\pi \hbar}{e^{2}} \frac{\left\langle R_{A}(E)\right\rangle}{\left\langle 1-R_{A}(E)\right\rangle},
$$

where the reflection probability $R_{A}(E)$ is given by

$$
R_{A}(E)=\left(\frac{m}{\hbar^{2} k_{A}}\right)^{2}\left|\int d z_{1} d z_{2} e^{i k_{A}\left(z_{1}+z_{2}\right)} \bar{T}_{A A}\left(z_{1}, z_{2}\right)\right|^{2} .
$$

The resistance under the weak-scattering limit $R_{s, \text { weak }}$ is expressed as

$$
R_{s, \text { weak }}=\frac{\pi \hbar}{e^{2}}\left\langle\left(\frac{m}{\hbar^{2} k_{A}}\right)^{2}\left|\int d z_{1} d z_{2} e^{i k_{A}\left(z_{1}+z_{2}\right)} \bar{T}_{A A}\left(z_{1}, z_{2}\right)\right|^{2}\right\rangle .
$$

We should notice that impurity scattering is treated non-perturbatively through the exact $T$-matrix in the above formulas. If the $T$-matrix in Eq. (14) is approximated with the lowest order: $\bar{T}_{A A}\left(z_{1}, z_{2}\right) \simeq \bar{V}_{A A}\left(z_{1}\right) \delta\left(z_{1}-z_{2}\right)$, the resistance $R_{s, \text { weak }}^{B}$ becomes

$$
R_{s, \text { weak }}^{B}=\frac{\pi \hbar}{e^{2}}\left\langle\left(\frac{m}{\hbar^{2} k_{A}}\right)^{2}\left|\int d z_{1} e^{i 2 k_{A} z_{1}} \bar{V}_{A A}\left(z_{1}\right)\right|^{2}\right\rangle,
$$

and this is usually referred to the Born approximation.

\section{Semiclassical approach: Boltzmann picture}

The BTE for nonequilibrium distribution $f\left(k, \varepsilon_{A}, z\right)$ under the nanowire structures is given by ${ }^{37,38}$

$$
\begin{aligned}
\frac{\partial f}{\partial t}+\dot{z} \frac{\partial f}{\partial z}+\dot{k} \frac{\partial f}{\partial k}= & \sum_{k^{\prime}, B} P\left(k^{\prime} B ; k A\right) f\left(k^{\prime}, \varepsilon_{B}, z\right)\left\{1-f\left(k, \varepsilon_{A}, z\right)\right\} \\
& -\sum_{k^{\prime}, B} P\left(k A ; k^{\prime} B\right) f\left(k, \varepsilon_{A}, z\right)\left\{1-f\left(k^{\prime}, \varepsilon_{B}, z\right)\right\} \\
= & \sum_{k^{\prime}, B} P\left(k^{\prime} B ; k A\right)\left\{f\left(k^{\prime}, \varepsilon_{B}, z\right)-f\left(k, \varepsilon_{A}, z\right)\right\},
\end{aligned}
$$

where $P\left(k^{\prime} B ; k A\right)$ is the transition probability per unit time from the eigenstate $\left(k^{\prime} B\right)$ to $(k A)$ of the unperturbed Hamiltonian $\hat{H}_{0}$. In the last equality, we have used the fact that $P\left(k^{\prime} B ; k A\right)=P\left(k A ; k^{\prime} B\right)$ for elastic scattering under the isotropic band structure. ${ }^{39}$ We now assume that the distribution function is approximated by a shifted equilibrium distribution under the locally uniform electric field $F$ such that

$$
f\left(k, \varepsilon_{A}, z\right) \approx f_{F D}\left(k, \varepsilon_{A}, z\right)-e \tau \frac{\hbar k}{m}\left(-\frac{\partial f_{F D}\left(k, \varepsilon_{A}, z\right)}{\partial E}\right) F,
$$

where $f_{F D}\left(k, \varepsilon_{A}, z\right)$ is the local equilibrium distribution at the axial position $z$ and given by the Fermi-Dirac distribution. The relaxation time $\tau$ is determined from the BTE and given ${ }^{40}$

$$
\frac{1}{\tau(k, A)}=\sum_{k^{\prime}, B} P\left(k A ; k^{\prime} B\right)\left(1-\frac{k^{\prime}}{k}\right) \text {. }
$$

The transition probability $P\left(k A ; k^{\prime} B\right)$ is calculated by Fermi's Golden rule

$$
P\left(k A ; k^{\prime} B\right)=\frac{2 \pi}{\hbar}\left|\left\langle\phi_{k^{\prime} B}|\hat{T}| \phi_{k A}\right\rangle\right|^{2} \delta\left(E-E^{\prime}\right),
$$

where $\hat{T}$ is the $T$-operator defined in Sec. II B associated with the scattering potential operator $\hat{V} . E$ and $E^{\prime}$ are the total energies of the electron before and after scattering, respectively, and they are given by $E=\hbar^{2} k^{2} /(2 m)+\varepsilon_{A}$ and $E^{\prime}=\hbar^{2} k^{\prime 2} /(2 m)+\varepsilon_{B}$.

The electric current $I$ at position $z$ is evaluated by

$$
I=-\frac{e}{\pi} \sum_{A} \int_{-\infty}^{\infty} d k \frac{\hbar k}{m} f\left(k, \varepsilon_{A}, z\right) \theta\left(E-\varepsilon_{A}\right)
$$

and we find that the impurity-limited resistance $R_{s}^{B T E}$ under the linear response regime is given by

$$
R_{s}^{B T E}=\frac{\pi \hbar}{e^{2}} \frac{1}{g_{s u b}} \frac{g_{s u b}}{\sum_{A}\left\langle\frac{2 v_{t h}\left(E, \varepsilon_{A}\right) \tau\left(E, \varepsilon_{A}\right) \theta\left(E-\varepsilon_{A}\right)}{L}\right\rangle},
$$

where $v_{t h}\left(E, \varepsilon_{A}\right)$ is the magnitude of electron velocity along the axis direction and given by $v_{t h}\left(E, \varepsilon_{A}\right)=\sqrt{2\left(E-\varepsilon_{A}\right) / m}$. Here, we have used the fact that the electric field $F$ at the axial position $z$ is approximated by the mean electric field in the channel region with no impurity and, thus, expressed by the potential drop $V$ divided by the channel length $L$. This is physically reasonable because the applied drain voltage is assumed to be small and the potential modulation caused by ionized impurities is just the screened short-range potential. Hence, the electrical current $I$ could be evaluated at any position $z$ in the channel, and $z$-dependence could be eliminated from the local equilibrium distribution $f_{F D}$. It should be noted that the equilibrium distribution $f_{F D}(E)$ in Eq. (31) is the equilibrium distribution inside the nanowire. Under the linear response regime, however, $f_{F D}(E)$ becomes identical to the equilibrium distribution $f_{F D}(E)$ in the source and drain regions. Therefore, thermal average represented by $\langle\cdots\rangle$ in Eq. (31) becomes identical to the one we have defined in the Landauer approach by Eq. (8).

Under the extreme quantum limit where only the lowest subband is involved in electron transport, $R_{s}^{B T E}$ is simplified as

$$
R_{s}^{B T E}=\frac{\pi \hbar}{e^{2}} \frac{L}{\left\langle 2 v_{t h}(E) \tau(E)\right\rangle} .
$$

Here, $A=(l=0, n=1)$ and, thus, the subband index $A$ has been eliminated from the arguments of $v_{t h}$ and $\tau$. The relaxation time $\tau(E)$ is calculated from the BTE with Eq. (28) and we obtain

$$
\frac{1}{\tau(E)}=\frac{2 m}{|k| \hbar^{3}} \frac{1}{L}\left|\int d z^{\prime} d z e^{i k\left(z^{\prime}+z\right)} \bar{T}_{A A}\left(z^{\prime}, z\right)\right|^{2} .
$$

Multiplying both sides of Eq. (33) by $\frac{\pi \hbar}{e^{2}} \frac{L}{2 v_{t h}(E)}$ and taking the thermal average as defined by Eq. (8), we find

$$
\frac{\pi \hbar}{e^{2}}\left\langle\frac{L}{2 v_{t h}(E) \tau(E)}\right\rangle=\frac{\pi \hbar}{e^{2}}\left\langle\left(\frac{m}{\hbar^{2} k}\right)^{2}\left|\int d z^{\prime} d z e^{i k\left(z^{\prime}+z\right)} \bar{T}_{A A}\left(z^{\prime}, z\right)\right|^{2}\right\rangle .
$$


This expression is identical to the impurity-limited resistance under the weak-scattering limit $R_{s, \text { weak }}$ in the Landauer approach, as given by Eq. (24). If electrons in the source and drain are highly degenerate, the derivative of the equilibrium distribution included in Eq. (8) has a peak structure around the Fermi energy $\mu_{L}$ and, thus, the energy spectrum of the incoming electrons becomes narrow unless temperature is very high. ${ }^{41}$ Therefore, we may relate Eq. (34) with $R_{s}^{B T E}$ such that

$$
\begin{aligned}
R_{s, \text { weak }} & =\frac{\pi \hbar}{e^{2}}\left\langle\frac{L}{2 v_{t h}(E) \tau(E)}\right\rangle \\
& \approx \frac{\pi \hbar}{e^{2}} \frac{L}{2 v_{t h}\left(\mu_{L}\right) \tau\left(\mu_{L}\right)} \\
& \approx \frac{\pi \hbar}{e^{2}} \frac{L}{\left\langle 2 v_{t h}(E) \tau(E)\right\rangle}=R_{s}^{B T E} .
\end{aligned}
$$

Since $R_{s, \text { weak }}$ is obtained by truncating the series with respect to $\left\langle R_{A}\right\rangle$ by the first term, Eq. (35) indeed confirms the fact that the BTE holds true only in the regime of the weak scattering limit (and the Born approximation). ${ }^{42}$ If scattering is strong, it is inevitable to employ the Landauer approach along with the exact $T$-matrix, i.e., impurity scattering is treated non-perturbatively and the denominator (the transmission probability) in $R_{s}$ is taken into account.

In addition, we would like to stress that in both approaches, the potential modulation over the whole channel region is treated as a single scattering potential and completely coherent. Therefore, the above claim that $R_{s}^{B T E}$ is nearly identical to $R_{s, \text { weak }}$ holds true only for short channel wire structures, under which the channel length is shorter than the incoherent scattering length so that no self-averaging is involved. In this case, we are able to obtain Landauer's results even from the BTE as far as the weak-scattering limit holds. In the long channel wires, on the other hand, the phase coherence is easily broken due to phase breaking processes such as phonon scattering, and thus, scattering with different impurities could be regarded as independent. This is equivalent to saying that the spatial average over the impurity configuration (self-averaging) is inevitably involved in long channel wires. Then, it is rather unrealistic to regard the potential modulation caused by many impurities over the whole channel region as a single scattering potential because the electron distribution would change its shape at each scattering center. ${ }^{43}$ Under the framework of the BTE, this is usually avoided by replacing the localized scattering potential by the space-averaged potential and multiplying the impurity density to the transition probability (not to the transition amplitude). In the case of scattering approaches, however, self-averaging seems to be possible only if phase-breaking scattering is explicitly included in the calculations.

To summarize, the impurity-limited resistance $R_{s}^{B T E}$ from the BTE approach in short channel nanowires becomes identical to $R_{s, \text { weak }}$ from the Landauer approach under the weak-scattering limit if the energy spectrum of the incoming electrons from the source and drain regions where electrons are highly degenerate is narrow, i.e., at low temperature.

\section{Landauer versus BTE in scattering-limited resistance}

The above results lead to another interesting observation. As the broadness in energy spectrum of the incoming electrons from the reservoirs is changed, we are able to distinguish the impurity-limited resistances obtained from the Landauer formula and that from the BTE.

Assuming that the scattering time $\tau(E)$ due to scattering is related with electron kinetic energy $E$ by

$$
\tau(E)=c_{0} E^{\eta},
$$

$R_{s}^{B T E}$ and $R_{S, \text { weak }}$ could be evaluated. Here, $c_{0}$ is constant and $\eta=3 / 2$ for impurity scattering, and the phonon scattering roughly corresponds to $\eta=1 / 2$ for $1 \mathrm{D}$ wire structures. ${ }^{44,45}$ Figure 2 shows the resistances as a function of the broadness of energy spectrum of the incoming electrons. The Fermi level $\mu_{L}$ of the source and drain is assumed to be higher than the bottom of the lowest subband of the nanowire. Both $R_{s}^{B T E}$ and $R_{s, \text { weak }}$ indeed coincide at small energy broadening. However, as the energy broadening becomes large, they begin to deviate. In the case of impurity scattering, $R_{s, \text { weak }}$ rises rapidly, whereas $R_{s}^{B T E}$ slightly decreases. Such a large difference in the resistance results from the energy dependence of the scattering time and peculiar to impurity scattering. For phonon scattering, the difference between $R_{s}^{B T E}$ and $R_{s, \text { weak }}$ is not so significant. Therefore, it would be very interesting to investigate experimentally the impurity-limited resistance under the weak-scattering regime to figure out which energy dependence is more realistic.

\section{Short-range scattering by localized impurities}

The formulas derived in Sec. II B are quite general; they could be applied to any type of elastic scattering potential to derive the transmission and reflection amplitudes.

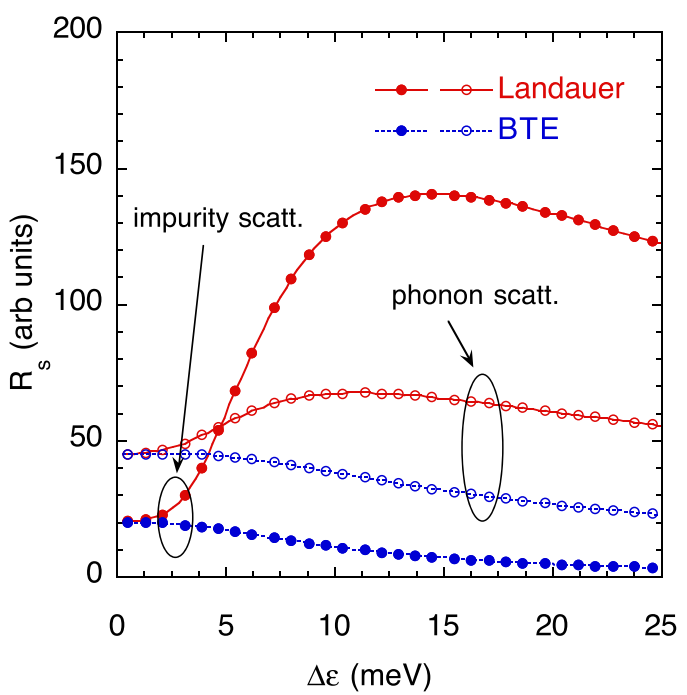

FIG. 2. Impurity-limited and phonon-limited resistance under the weakscattering regime as a function of the broadness of energy spectrum of the in-coming electrons. $R_{s, \text { weak }}$ obtained from the Landauer approach is plotted with red lines and $R_{s}^{B T E}$ from the BTE approach is plotted by blue lines. The cases of impurity scattering $(\eta=3 / 2)$ and phonon scattering $(\eta=1 / 2)$ are shown with solid and empty symbols, respectively. 
Here, in order to pursue the analytical means as much as possible and to make the physics involved clearer, we apply the formulas for a very simple, yet fruitful case; the scattering potential of ionized impurities is given by the localized $\delta$ function. This corresponds to the extreme case in which the screening is so strong that the long-range part of the impurity scattering potential is completely suppressed.

Suppose that there are $N_{\text {imp }}$ impurities doped in the channel region and the scattering potential is given in the coordinate space by

$$
V(\mathbf{R})=\sum_{r=1}^{N_{i m p}}\left(v_{c} a S\right) \delta^{(3)}\left(\mathbf{R}-\mathbf{R}_{0 r}\right),
$$

where $a$ represents the characteristic length along the wire axis direction over which the scattering potential is effective and $S$ is the cross-sectional area of the wire. $v_{c}$ is the scattering potential energy and assumed to be constant for simplicity. ${ }^{46}$ The position vector $\mathbf{R}_{0 r}$ of the $r$-th impurity in the cylindrical coordinates is denoted by $\mathbf{R}_{0 r}=\left(\mathbf{r}_{0 r}, z_{0 r}\right)$ $=\left(r_{0 r}, \varphi_{0 r}, z_{0 r}\right)$. It should be noted that under the present theoretical framework, the impurity density is determined by the number of impurities $N_{i m p}$ in Eq. (37) and by the normalization constant $1 / \sqrt{L}$ of the subband wavefunction along the wire axis direction in Eq. (2). Since the impurity density in the channel region is assumed to be constant in this study, the channel length $L$ varies according to the number of impurities $N_{\text {imp }}$ doped in the channel. In addition, the electron density is assumed to be the same as the impurity density, to be consistent with the flat potential assumption in the channel region. In fact, the electron density dependence of the average impurity-limited resistance is of great importance from the application standpoint. However, for this purpose, it is essential to include the self-consistent potential by solving the Poisson equation, and thus, this part is left for future study.

The integral $I_{B A}^{\mp}$ including the reduced $T$-matrix in Eqs. (16) and (17) can be expanded as

$$
I_{B A}^{\mp}=I_{B A}^{(1)}+I_{B A}^{(2)}+I_{B A}^{(3)}+\cdots .
$$

In order to clarify the structure inherent in $I_{B A}^{\mp}$, we introduce the following shorthand notations:

$$
g_{r s}^{I}=-i \frac{m}{\hbar^{2} k_{I}} e^{i k_{I}\left|\Delta_{r s}\right|}=g_{0}^{I} e^{i k_{I}\left|\Delta_{r s}\right|} \equiv\left\langle I\left|\hat{g}_{r s}\right| I\right\rangle,
$$

where $\hat{g}_{r s}=\hat{g}_{0} e^{i \hat{k}\left|\Delta_{r s}\right|}$ with $\Delta_{r s}=z_{0 r}-z_{0 s} .|I\rangle$ represents the eigenstate of the subband $I$ in the nanowire. Also, we define

$$
\Xi_{I J}^{r}=\left(v_{c} a S\right) \xi_{I}^{*}\left(\mathbf{r}_{0 r}\right) \xi_{J}\left(\mathbf{r}_{0 r}\right) \equiv\left\langle I\left|\hat{\Xi}^{r}\right| J\right\rangle .
$$

Then, the reduced scattering potential $\bar{V}_{B A}(z)$ defined by Eq. (15) is simply written as

$$
\bar{V}_{B A}(z)=\sum_{r=1}^{N_{i m p}} \Xi_{B A}^{r} \delta\left(z-z_{0 r}\right) .
$$

The first term $I_{B A}^{(1)}$ is expressed as

$$
\begin{aligned}
I_{B A}^{(1)} & =\sum_{r=1}^{N_{i m p}} e^{\mp i k_{B} z_{0 r}} g_{0}^{B} \Xi_{B A}^{r} e^{i k_{A} z_{0 r}} \\
& =\left\langle B\left|\sum_{r=1}^{N_{i m p}} e^{\mp i \hat{k}_{0 r}} \hat{g}_{0} \hat{\Xi}^{r} e^{i \hat{k} z_{0 r}}\right| A\right\rangle .
\end{aligned}
$$

Similarly, the second and third terms, $I_{B A}^{(2)}$ and $I_{B A}^{(3)}$, are given by

$$
\begin{aligned}
I_{B A}^{(2)} & =\sum_{r, s=1}^{N_{i m p}} e^{\mp i k_{B} z_{0 r}} g_{0}^{B} \sum_{C} \Xi_{B C}^{r} g_{r s}^{C} \Xi_{C A}^{s} e^{i k_{A} z_{0 s}} \\
& =\left\langle B\left|\sum_{r, s=1}^{N_{i m p}} e^{\mp i \hat{k} z_{0 r}} \hat{g}_{0} \hat{\Xi}^{r} \hat{g}_{r s} \hat{\Xi}^{s} e^{i \hat{k} z_{0 s}}\right| A\right\rangle
\end{aligned}
$$

and

$$
\begin{aligned}
I_{B A}^{(3)} & =\sum_{r, t, s=1}^{N_{\text {imp }}} e^{\mp i k_{B} z_{0 r}} g_{0}^{B} \sum_{C, D} \Xi_{B C}^{r}\left(g_{r t}^{C} \Xi_{C D}^{t} g_{t s}^{D}\right) \Xi_{D A}^{s} e^{i k_{A} z_{0 s}} \\
& =\left\langle B\left|\sum_{r, t, s=1}^{N_{i m p}} e^{\mp i \hat{k} z_{0 r}} \hat{g}_{0} \hat{\Xi}^{r}\left(\hat{g}_{r t} \hat{\Xi}^{t} \hat{g}_{t s}\right) \hat{\Xi}^{s} e^{i \hat{k} z_{0 s}}\right| A\right\rangle .
\end{aligned}
$$

Consequently, each term in Eq. (38) could be compactly rewritten by employing the matrix representation with respect to the position indices of the impurities. We obtain

$$
\begin{aligned}
& I_{B A}^{\mp}=\langle B|\left(\begin{array}{llll}
e^{\mp i \hat{k} z_{01}} & e^{\mp i \hat{k} z_{02}} & \cdots & e^{\mp i \hat{k} z_{0 N} i m p}
\end{array}\right) \\
& \times \hat{g}_{0} \hat{\Xi}\left(\frac{1}{1-\hat{g} \hat{\Xi}}\right)\left(\begin{array}{c}
e^{i \hat{k} z_{01}} \\
e^{i \hat{k} z_{02}} \\
\vdots \\
e^{i \hat{k} z_{0 N i m p}}
\end{array}\right)|A\rangle
\end{aligned}
$$

where we have defined

$$
\hat{\Xi}=\left(\begin{array}{cccc}
\hat{\Xi}^{1} & 0 & 0 & 0 \\
0 & \hat{\Xi}^{2} & 0 & 0 \\
0 & 0 & \ddots & 0 \\
0 & 0 & 0 & \hat{\Xi}^{N_{i m p}}
\end{array}\right)
$$

and

$$
\hat{g}=\hat{g}_{0}\left(\begin{array}{cccc}
1 & e^{i \hat{k}\left|\Delta_{12}\right|} & \cdots & e^{i \hat{k}\left|\Delta_{1 N_{i m p}}\right|} \\
e^{i \hat{k}\left|\Delta_{21}\right|} & 1 & \cdots & e^{i \hat{k}\left|\Delta_{2 N_{i m p}}\right|} \\
\vdots & \vdots & \ddots & \vdots \\
e^{i \hat{k}\left|\Delta_{N_{i m p}}\right|} & e^{i \hat{k}\left|\Delta_{N_{i m p}}\right|} & \cdots & 1
\end{array}\right) .
$$

Since no approximation has been made so far, Eq. (45) is exact. Under the extreme quantum limit where only the lowest subband $A$ is involved, the operators $\hat{g}$ and $\hat{\Xi}$ commute each other and the resulting expressions are used in the following numerical calculations. 


\section{NUMERICAL CALCULATIONS AND DISCUSSION}

\section{A. Single-impurity under extreme quantum limit}

Let us consider the simplest case; there is only one impurity doped in the nanowire. ${ }^{24,26,47}$ Employing Eq. (45) under the extreme quantum limit, the transmission and reflection amplitudes are, respectively, given by

$$
t_{A A}(E)=1+I_{A A}^{-}=\frac{1}{1+i \gamma\left(E, r_{01}\right)}
$$

and

$$
r_{A A}(E)=I_{A A}^{+}=-\frac{i \gamma\left(E, r_{01}\right)}{1+i \gamma\left(E, r_{01}\right)} e^{i 2 k_{A} z_{01}},
$$

where the scattering parameter $\gamma\left(E, r_{01}\right)(\in \mathbb{R})$ for the incoming electrons with total energy $E\left(\geq \varepsilon_{A}\right)$ scattered by the impurity at the radial position $r_{01}$ is defined by

$$
\gamma\left(E, r_{01}\right)=i g_{0}^{A} \Xi_{A A}^{1}=v_{c} \frac{a}{\hbar} \sqrt{\frac{m}{2\left(E-\varepsilon_{A}\right)}} S\left|\xi_{A}\left(r_{01}\right)\right|^{2} .
$$

The exact impurity-limited resistance $R_{s}\left(r_{01}\right)$ associated with the single-impurity at $r_{01}$ is calculated from Eq. (22) and given by

$$
R_{S}\left(r_{01}\right)=\frac{\pi \hbar}{e^{2}} \frac{\left\langle R_{A}\right\rangle}{\left\langle T_{A}\right\rangle}=\frac{\pi \hbar}{e^{2}} \frac{\left\langle\frac{\gamma\left(E, r_{01}\right)^{2}}{1+\gamma\left(E, r_{01}\right)^{2}}\right\rangle}{\left\langle\frac{1}{1+\gamma\left(E, r_{01}\right)^{2}}\right\rangle} .
$$

The resistance under the weak-scattering limit $R_{s, \text { weak }}$ is calculated from (24) and given by

$$
R_{s, \text { weak }}\left(r_{01}\right)=\frac{\pi \hbar}{e^{2}}\left\langle R_{A}\right\rangle=\frac{\pi \hbar}{e^{2}}\left\langle\frac{\gamma\left(E, r_{01}\right)^{2}}{1+\gamma\left(E, r_{01}\right)^{2}}\right\rangle
$$

and the resistance under the Born approximation is calculated from (25) and given by

$$
R_{s, \text { weak }}^{B}\left(r_{01}\right)=\frac{\pi \hbar}{e^{2}}\left\langle\gamma\left(E, r_{01}\right)^{2}\right\rangle .
$$

Notice that the restriction imposed on the scattering parameter, $\left|\gamma\left(E, r_{0}\right)\right|<1$, by the condition that the Born series should be convergent is removed in Eqs. (51) and (52), in which impurity scattering is treated non-perturbatively. Also, $R_{s}$ is not bounded above, whereas $R_{s, \text { weak }}$ and $R_{s, \text { weak }}^{B}$ are bounded. We notice that $R_{s, \text { weak }}$ is somewhat inconsistent with the approximations employed in its derivation since $R_{S}$ is truncated by the first term with $\left\langle R_{A}\right\rangle$. Roughly speaking, this is equivalent to ignoring the vertex corrections in the conductivity calculation, and this point will be discussed elsewhere. Of course, both $R_{s, \text { weak }}$ and $R_{s, \text { weak }}^{B}$ approach the exact result $R_{s}$ when $|\gamma| \ll 1$.

We should also point out that the impurity-limited resistance at $T=0$ reduces to

$$
R_{S}\left(r_{01}\right) \underset{T=0}{\rightarrow} \frac{\pi \hbar}{e^{2}}\left\langle\gamma\left(E, r_{01}\right)^{2}\right\rangle=\frac{\pi \hbar}{e^{2}} \gamma\left(\mu_{L}, r_{01}\right)^{2},
$$

assuming that the Fermi energy of the reservoirs is above the bottom of the lowest subband of the nanowire, $\mu_{L}>\varepsilon_{A}$. This expression becomes identical to $R_{s, \text { weak }}^{B}$, and thus, the Born approximation, rather than $R_{s, \text { weak }}$ in which impurity scattering is treated nonperturbatively, becomes exact at very low temperature.

\section{Scattering strength}

We now estimate the magnitude of the scattering parameter $\gamma\left(E, r_{01}\right)$ under the quasi-1D nanowires. Assuming that the impurity potential $V_{s c}(\mathbf{R})$ is given by the Yukawa potential with the screening length $\lambda_{s c}$ such that

$$
V_{s c}(\mathbf{R})=\frac{e}{4 \pi \varepsilon_{s}} \frac{e^{-\frac{R}{2 s c}}}{R}
$$

where $\varepsilon_{s}$ is the dielectric constant of the semiconductor substrate of the nanowire, we could estimate the scattering potential energy $v_{c}$ as

$$
\left|v_{c}\right| \approx \frac{e}{\Omega_{s c}} \int d^{3} R V_{s c}(\mathbf{R}) \sim 0.36609 \frac{1}{\lambda_{s c}(\mathrm{~nm})}(\mathrm{eV}),
$$

where $\Omega_{s c}$ is the volume of the sphere with radius $\lambda_{s c}$. Hence, the magnitude of the scattering parameter is approximately given by ${ }^{48}$

$$
\left|\gamma\left(E, r_{01}\right)\right| \sim 16.6365 \times \frac{a}{\lambda_{s c} \sqrt{E-\varepsilon_{A}(\mathrm{meV})}},
$$

where we have assumed that $S\left|\xi_{A}\left(r_{01}\right)\right|^{2} \sim 1$.

Since the Coulomb potential due to ionized impurities is confined in a very limited region under the gated nanodevice structure, $\left|v_{c}\right|$ tends to be larger as the structure shrinks. In addition, the lowest subband wave-function has a peak on the wire axis so that the effective scattering potential weighted with the wave-function is enhanced as the size of the wire cross-section shrinks. Consequently, $|\gamma|$ becomes larger than unity, and the weak-scattering limit easily breaks down in the nanowire. It is, thus, expected that both the higher-order corrections of impurity scattering and the denominator of the impurity-limited resistance in Eq. (51) should be taken into account.

\section{Radial position dependence of resistance}

Figure 3 shows the impurity-limited resistances at $T=300$ and $30 \mathrm{~K}$ as a function of the radial position of the single impurity in the cylindrical wire with $r_{s}=2 \mathrm{~nm}$. The scattering potential energy $v_{c}$ is assumed to be $v_{c}=183 \mathrm{meV}$, corresponding to the screening length of $\lambda_{s c}=2 \mathrm{~nm}$. The contact (quantum) resistance $R_{0}$ is given by $R_{0}=\pi \hbar / e^{2}$. The numerical values of the parameters employed are summarized in Table I.

At room temperature, $R_{s, \text { weak }}$ and $R_{s, \text { weak }}^{B}$ largely deviate from the exact result $R_{s}$, and thus, the weak-scattering limit indeed breaks down. Especially, $R_{s, \text { weak }}$ is always bounded 

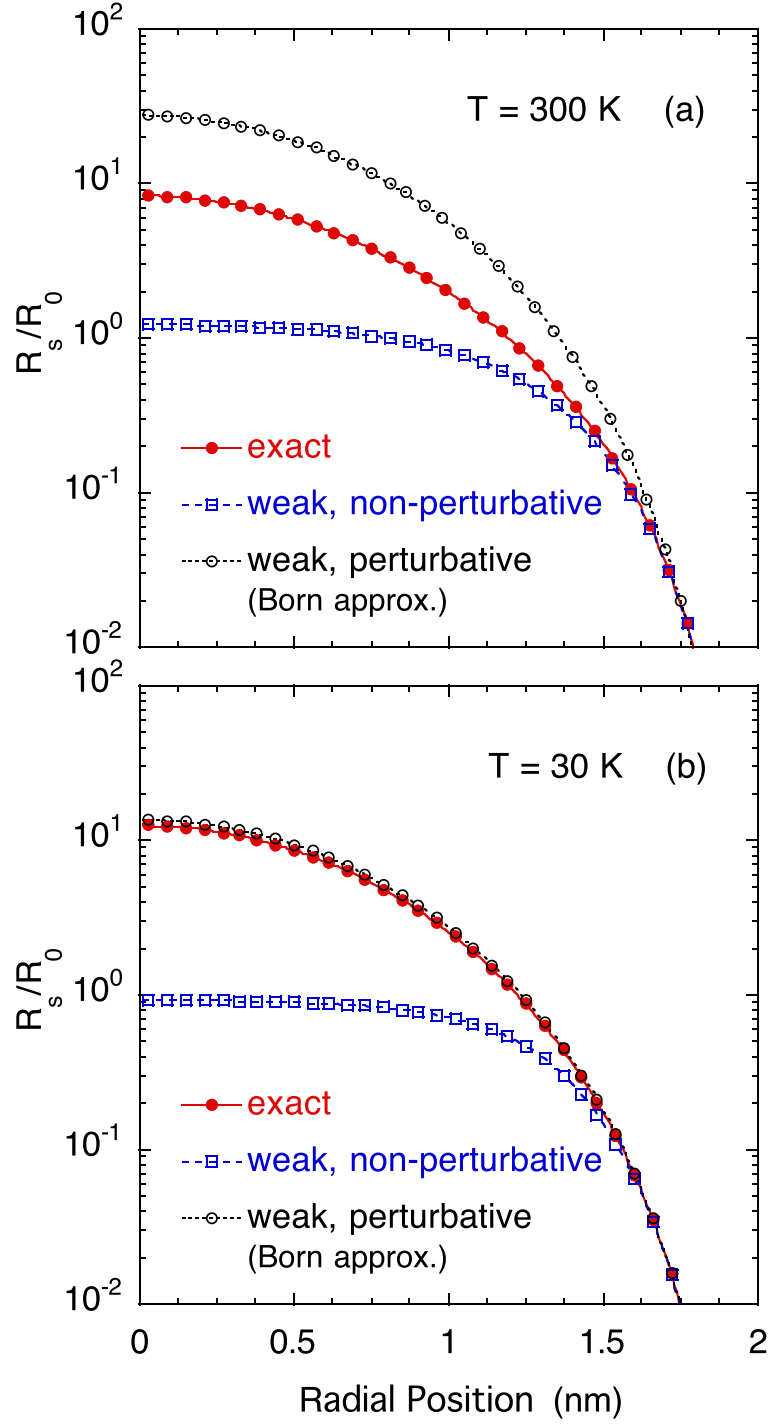

FIG. 3. Impurity-limited resistance as a function of the radial position of the single impurity in the cylindrical wire for (a) $T=300$ and (b) $30 \mathrm{~K}$. The scattering potential energy is $v_{c}=183 \mathrm{meV}$. The resistances from the exact formula of $R_{S}$ (red solid line), and from the two approximations, $R_{s, \text { weak }}$ (blue dashed line) and $R_{s, \text { weak }}^{B}$ (black dotted line), are shown.

by the upper limit of the reflection probability (due to the non-perturbative treatment of impurity scattering) and underestimates the impurity-limited resistance at any temperature. It is also clear that the variation of the resistance with respect to the radial location of the impurity results from the position-dependence of the subband wave-function $\left|\xi_{A}\left(r_{0}\right)\right|$, which also has a maximum on the wire axis and decreases

TABLE I. List of parameters.

\begin{tabular}{lcc}
\hline \hline$r_{s}$ & Radius of wire & $2 \mathrm{~nm}$ \\
$a$ & Characteristic length & $0.5 \mathrm{~nm}$ \\
$S\left(=\pi r_{s}^{2}\right)$ & Cross-sectional area & $12.6 \mathrm{~nm}^{2}$ \\
$n_{e l}$ & Electron density & $2 \times 10^{19} \mathrm{~cm}^{-3}$ \\
$L$ & Channel length (single impurity) & $3.98 \mathrm{~nm}$ \\
& Channel length (two impurities) & $7.96 \mathrm{~nm}$ \\
$m$ & Effective mass & $0.315 \mathrm{~m}_{0}$ \\
$\varepsilon_{s}$ & Dielectric constant (wire) & $11.8 \varepsilon_{0}$ \\
\hline \hline
\end{tabular}

along the radial direction. In reality, the impurity scattering potential is greatest on the wire axis and gets weaker in the radial direction toward the interface of the gate oxide. The present results, though the scattering potential is constant and no long-range part of the potential is included, are consistent with the previous results of the tight-binding NEGF simulations with more realistic scattering potential. ${ }^{24,26}$ Furthermore, we notice that as temperature is lowered, the Born approximation becomes close to the exact result although the scattering parameter $|\gamma|$ exceeds unity above which the weak scattering limit (the Born approximation) breaks down. This is consistent with Eq. (54).

These results confirm the conjecture mentioned above: The higher order corrections associated with the multiple scattering with the same impurity are significant at room temperature in short-channel nanowires. Thus, in general, the non-perturbative treatment for the transition matrix as well as the inclusion of the denominator $\left\langle T_{A}\right\rangle$ in $R_{S}$ is crucial under the nanowire structures.

\section{Ensemble average resistance over impurity location}

The meaning of spatial average and the variations of $R_{s}$ are rather trivial in the single-impurity case. Averaging $R_{s}$ in the coordinate space simply implies that the impurity location is averaged over the cross-sectional area, irrespective of the wire axis direction. The probability density of the impurity-limited resistance $F\left(R_{S}\right)$ is calculated from

$$
F\left(R_{s}\right)=\int d^{3} \mathbf{R}_{01} \delta\left(R_{s}-R_{s}\left(\mathbf{R}_{01}\right)\right) p_{i m p}\left(\mathbf{R}_{01}\right),
$$

where $p_{\text {imp }}\left(\mathbf{R}_{01}\right)$ is the probability density of the impurity position $\mathbf{R}_{01}$. If the impurity is distributed uniformly over the volume of the channel region, the probability density becomes constant and $F\left(R_{s}\right)$ becomes

$$
F\left(R_{s}\right)=\frac{2}{r_{s}^{2}} \int_{0}^{r_{s}} d r_{01} r_{01} \delta\left(R_{s}-R_{s}\left(r_{01}\right)\right) .
$$

The probability density $F\left(R_{s}\right)$ calculated from Eq. (59) is shown in Fig. 4 as a function of $R_{s} . F\left(R_{s}\right)$ has a peak at very low resistance, which comes from the resistance near the edge of the nanowire where the subband wave-function almost vanishes, whereas the peak at high resistance is due to the contribution from the wire axis where the subband wavefunction has a peak. Therefore, $R_{s}$ is dominantly determined by the resistance near the interface with the gate-oxide if the spatial configuration of impurities is uniform. This trend is expected to hold true even stronger if the impurity potential is more realistic because the impurity potential is strongly screened by the surrounding materials near the interface.

The average impurity-limited resistance $\bar{R}_{s}$ under the uniform impurity distribution is obtained from

$$
\bar{R}_{s}=\int_{0}^{\infty} d R_{s} R_{s} F\left(R_{s}\right)=\frac{2}{r_{s}^{2}} \int_{0}^{r_{s}} d r_{01} r_{01} R_{s}\left(r_{01}\right) .
$$

Figure 5 shows $\bar{R}_{s}$ as a function of the scattering potential energy $v_{c}$ at $T=300$ and $30 \mathrm{~K} . \bar{R}_{s}$ calculated from the exact 


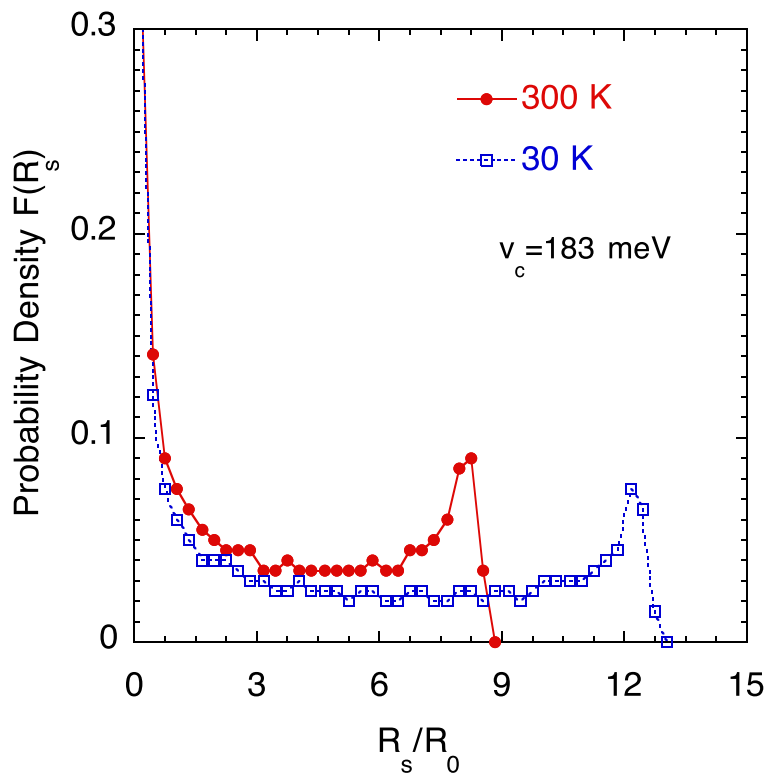

FIG. 4. Probability density $F\left(R_{S}\right)$ of the impurity-limited resistance as a function of resistance $R_{s}$ at $T=300 \mathrm{~K}$ (red solid line) and $30 \mathrm{~K}$ (blue dotted line).

formula of $R_{S}$ and from the two approximations of $R_{s, \text { weak }}$ and $R_{s \text {,weak }}^{B}$ are also shown. As expected from the probability density $F\left(R_{S}\right)$, the average resistance $\bar{R}_{S}$ is much smaller than the resistance of the single-impurity located on the wire axis. Hence, $\bar{R}_{s}$ obtained from the Born approximation $R_{s, \text { weak }}^{B}$ becomes very close to the exact $\bar{R}_{s}$. At lower temperature $(T=30 \mathrm{~K})$, these two average resistances nearly coincide. On the other hand, $\bar{R}_{s}$ obtained from the weak-scattering limit $R_{\text {s,weak }}$, in which the scattering is treated nonperturbatively, is very poor unless the scattering potential energy is very small. These results imply that unless the impurity density is extremely high, the Born approximation should work quite well for the ensemble average resistance of shortchannel nanowires or the resistance of long-channel nanowires where self-averaging of the impurity configuration inside the nanowire is implicitly involved. If the impurity density is high, the multiple-scattering with different impurities could affect the transport properties, as we shall discuss below.

\section{B. Two correlated impurities under extreme quantum limit}

Let us consider the case where two localized impurities are doped in the channel region so that the phase interference among the impurities at different sites comes into play.

As before, we consider the extreme quantum limit where only the lowest subband is involved in electron transport and we introduce the scattering parameter $\gamma_{r}\left(E, \mathbf{r}_{0 r}\right)(\in \mathbb{R})$ for the $r$-th impurity $(r=1,2)$ at position $\mathbf{R}_{0 r}=\left(\mathbf{r}_{0 r}, z_{0 r}\right)$ by

$$
\gamma_{r}\left(E, \mathbf{r}_{0 r}\right)=v_{c} \frac{a}{\hbar} \sqrt{\frac{m}{2\left(E-\varepsilon_{A}\right)}} S\left|\xi_{A}\left(\mathbf{r}_{0 r}\right)\right|^{2} .
$$

Hereafter, we eliminate the subscript $A$ in $k_{A}$, etc. Also, we assume that the relative position of the two impurities along
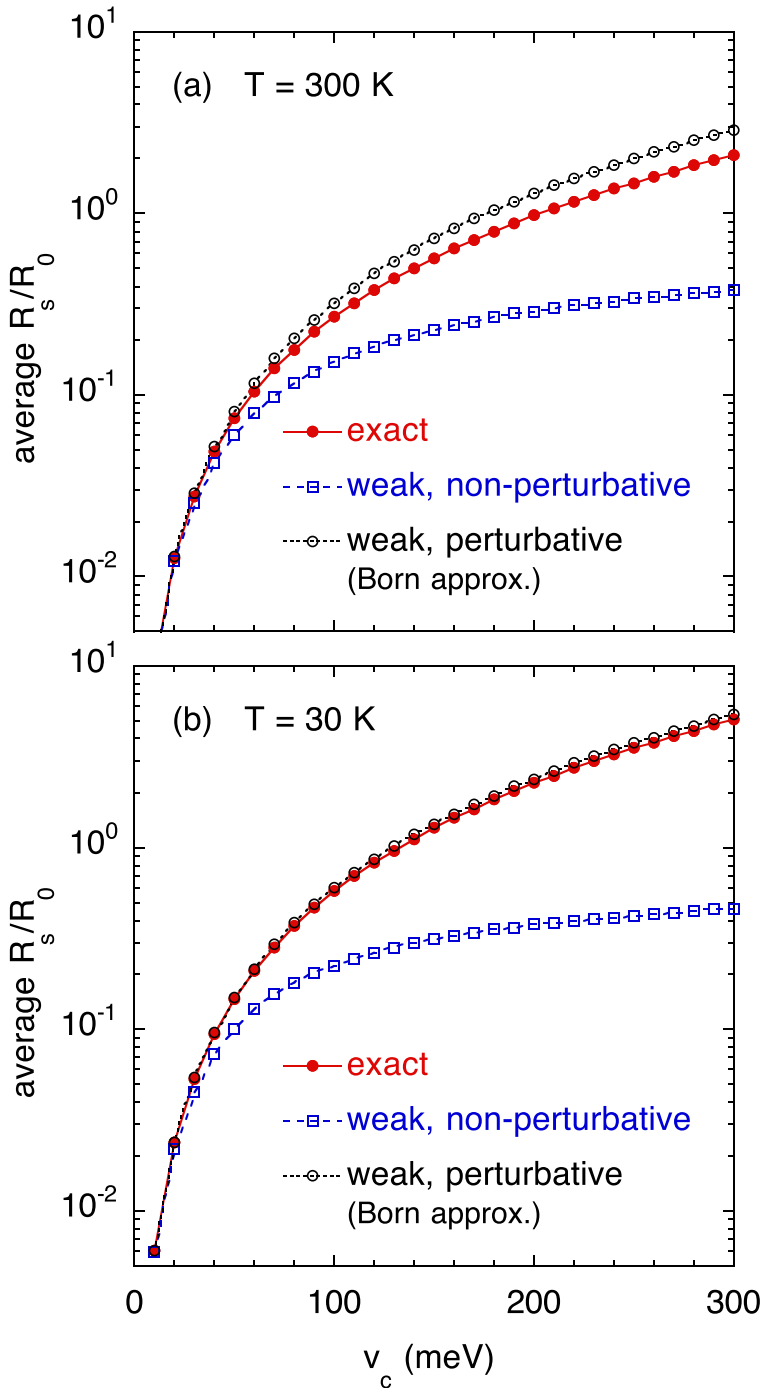

FIG. 5. Space-averaged impurity-limited resistance $\bar{R}_{s}$ as a function of the scattering potential energy $v_{c}$ for (a) $T=300$ and (b) $30 \mathrm{~K}$. The average resistances from the exact formula of $R_{S}$ (red solid line), and from the two approximations, $R_{s, \text { weak }}$ (blue dashed line) and $R_{s, \text { weak }}^{B}$ (black dotted line), are shown.

the wire axis direction satisfies $\Delta \equiv \Delta_{12} \geq 0$ since there is no essential difference in the axial position of two impurities. The integral $I_{A A}^{-}$given by Eq. (45) is expressed as

$$
I_{A A}^{-}=-\frac{i\left(\gamma_{1}+\gamma_{2}\right)+\gamma_{1} \gamma_{2}\left(e^{i 2 k \Delta}-1\right)}{1+i\left(\gamma_{1}+\gamma_{2}\right)+\gamma_{1} \gamma_{2}\left(e^{i 2 k \Delta}-1\right)},
$$

and the transmission probability $T_{A}(E)$ becomes

$$
T_{A}(E)=\left|1+I_{A A}^{-}\right|^{2}
$$

Similarly, the integral $I_{A A}^{+}$given by Eq. (45) is expressed as

$$
I_{A A}^{+}=e^{i 2 k z_{02}} \frac{\gamma_{2}\left(\gamma_{1}-i\right)-e^{i 2 k \Delta} \gamma_{1}\left(\gamma_{2}+i\right)}{1+i\left(\gamma_{1}+\gamma_{2}\right)+\gamma_{1} \gamma_{2}\left(e^{i 2 k \Delta}-1\right)},
$$

and the explicit expression of the reflection probability $R_{A}(E)$ is given by 


$$
R_{A}(E)=\left|I_{A A}^{+}\right|^{2}=\frac{\gamma_{1}^{2}+\gamma_{2}^{2}+2 \gamma_{1}^{2} \gamma_{2}^{2}+2 \gamma_{1} \gamma_{2}\left\{\left(1-\gamma_{1} \gamma_{2}\right) \cos (2 k \Delta)+\left(\gamma_{1}+\gamma_{2}\right) \sin (2 k \Delta)\right\}}{1+\gamma_{1}^{2}+\gamma_{2}^{2}+2 \gamma_{1}^{2} \gamma_{2}^{2}+2 \gamma_{1} \gamma_{2}\left\{\left(1-\gamma_{1} \gamma_{2}\right) \cos (2 k \Delta)+\left(\gamma_{1}+\gamma_{2}\right) \sin (2 k \Delta)\right\}} .
$$

Notice that the above expression is symmetric with respect to $\gamma_{1}$ and $\gamma_{2}$, as it should be. Also, no approximation has been made to derive the above expressions, and thus, we could easily confirm the fact that the electron flux is conserved

$$
T_{A}(E)+R_{A}(E)=1 .
$$

The exact impurity-limited resistance $R_{S}$ of two impurities is then obtained by

$$
R_{S}\left(\mathbf{r}_{01}, \mathbf{r}_{02}, \Delta\right)=\frac{\pi \hbar}{e^{2}} \frac{\left\langle R_{A}(E)\right\rangle}{\left\langle 1-R_{A}(E)\right\rangle} .
$$

Following the arguments for the single-impurity case, two approximations, $R_{s, \text { weak }}\left(\mathbf{r}_{01}, \mathbf{r}_{02}, \Delta\right)$ and $R_{s, \text { weak }}^{B}\left(\mathbf{r}_{01}, \mathbf{r}_{02}, \Delta\right)$, are expressed by

$$
R_{s, \text { weak }}\left(\mathbf{r}_{01}, \mathbf{r}_{02}, \Delta\right)=\frac{\pi \hbar}{e^{2}}\left\langle R_{A}(E)\right\rangle
$$

and

$$
R_{s, \text { weak }}^{B}\left(\mathbf{r}_{01}, \mathbf{r}_{02}, \Delta\right)=\frac{\pi \hbar}{e^{2}}\left\langle\Gamma_{A}(E)\right\rangle,
$$

where $\Gamma_{A}(E)$ is given by the numerator of Eq. (65).

\section{Coherent and incoherent limits}

Let us consider the two extreme cases: the most coherent and incoherent cases.

Under the most coherent case, in which the axial separation between two impurities diminishes $(\Delta \rightarrow 0)$, the transmission and reflection probabilities reduce to the following expressions:

$$
T_{A}(E)=\frac{1}{1+\left(\gamma_{1}+\gamma_{2}\right)^{2}}
$$

and

$$
R_{A}(E)=\frac{\left(\gamma_{1}+\gamma_{2}\right)^{2}}{1+\left(\gamma_{1}+\gamma_{2}\right)^{2}}
$$

Equations (70) and (71) exactly coincide with those of the single-impurity case if one replaces the scattering parameter $\gamma_{1}+\gamma_{2}$ by $n_{i m p} \gamma$, where $n_{i m p}$ is the number density of impurity. Roughly speaking, the resistance $R_{S}$ is proportional to the ratio of $R_{A}$ to $T_{A}$, and thus, $R_{S}$ could be approximated as

$$
R_{s} \sim\left(\gamma_{1}+\gamma_{2}\right)^{2} \rightarrow R_{s} \propto n_{i m p}^{2} \gamma^{2} .
$$

That is, $R_{s}$ becomes proportional to the square of $n_{i m p}$ and is strongly enhanced by the constructive phase interference among the impurities.
On the other hand, in the incoherent case where the phase interference between the impurities is ignored, the terms including the trigonometric functions in Eqs. (63) and (65) vanish and we obtain

$$
T_{A}(E)=\frac{1}{1+\left(\gamma_{1}^{2}+\gamma_{2}^{2}+2 \gamma_{1}^{2} \gamma_{2}^{2}\right)} \approx \frac{1}{1+\left(\gamma_{1}^{2}+\gamma_{2}^{2}\right)}
$$

and

$$
R_{A}(E)=\frac{\gamma_{1}^{2}+\gamma_{2}^{2}+2 \gamma_{1}^{2} \gamma_{2}^{2}}{1+\left(\gamma_{1}^{2}+\gamma_{2}^{2}+2 \gamma_{1}^{2} \gamma_{2}^{2}\right)} \approx \frac{\gamma_{1}^{2}+\gamma_{2}^{2}}{1+\left(\gamma_{1}^{2}+\gamma_{2}^{2}\right)},
$$

where higher order terms with respect to $\gamma_{1}$ and $\gamma_{2}$ are ignored. Again, Eqs. (73) and (74) coincide with those of the single-impurity case if one replaces the scattering parameter $\gamma_{1}^{2}+\gamma_{2}^{2}$ by $n_{\text {imp }} \gamma^{2}$. Therefore, the resistance $R_{s}$ becomes

$$
R_{s} \sim\left(\gamma_{1}^{2}+\gamma_{2}^{2}\right) \rightarrow R_{s} \propto n_{i m p} \gamma^{2},
$$

and $R_{s}$ is now proportional to $n_{i m p}$. Therefore, each impurity behaves as an independent scattering center, and phase interference plays no role. We are, therefore, able to recover the classical Ohm's law under the incoherent limit. ${ }^{49}$

\section{Phase interference between impurities on wire axis}

The impurity-limited resistance of the nanowire with two impurities doped in the channel is now evaluated numerically. For simplicity, two impurities are assumed to reside on the wire axis, so that $\gamma_{0}(E) \equiv \gamma_{1}\left(E, r_{01}=0\right)$ $=\gamma_{2}\left(E, r_{02}=0\right)$. The transmission and reflection probabilities are greatly simplified and the impurity-limited resistances under various approximations are evaluated with Eqs. (67), (68), and (69).

Figure 6 shows the impurity-limited resistance $R_{s}(\Delta)$ at $T=300$ and $30 \mathrm{~K}$ as a function of the impurity separation $\Delta$ along the wire axis direction. The scattering potential energy is set at $v_{c}=183 \mathrm{meV}$, corresponding to the screening length of $\lambda_{s c}=2 \mathrm{~nm}$. The results from the exact formula $R_{s}$ and two approximations, $R_{s, \text { weak }}$ and $R_{s, \text { weak }}^{B}$, are shown. The horizontal dashed line shows twice of $R_{s}^{\text {single }}$, where $R_{s}^{\text {single }}$ is the impurity-limited resistance obtained from the singleimpurity located at $r_{01}=0$. It is clear that both $R_{s, \text { weak }}$ and $R_{s, \text { weak }}^{B}$ break down and a large oscillatory behavior in the exact $R_{s}$ is observed in the first few $\mathrm{nm}$ for $T=300 \mathrm{~K}$. This oscillation becomes much clearer as temperature is lowered. This results from the trigonometric dependence in $R_{S}$ and caused by electron's phase interference among the two impurities. However, the oscillation rapidly damps at $T=300 \mathrm{~K}$ and $R_{s}$ approaches $2 R_{s}^{\text {single }}$. Since no averaging with respect to the configuration of impurities or phaserandomizing scattering is involved in the present calculations, this phase randomization is caused purely by the broadness of the energy spectrum of the in-coming electrons 

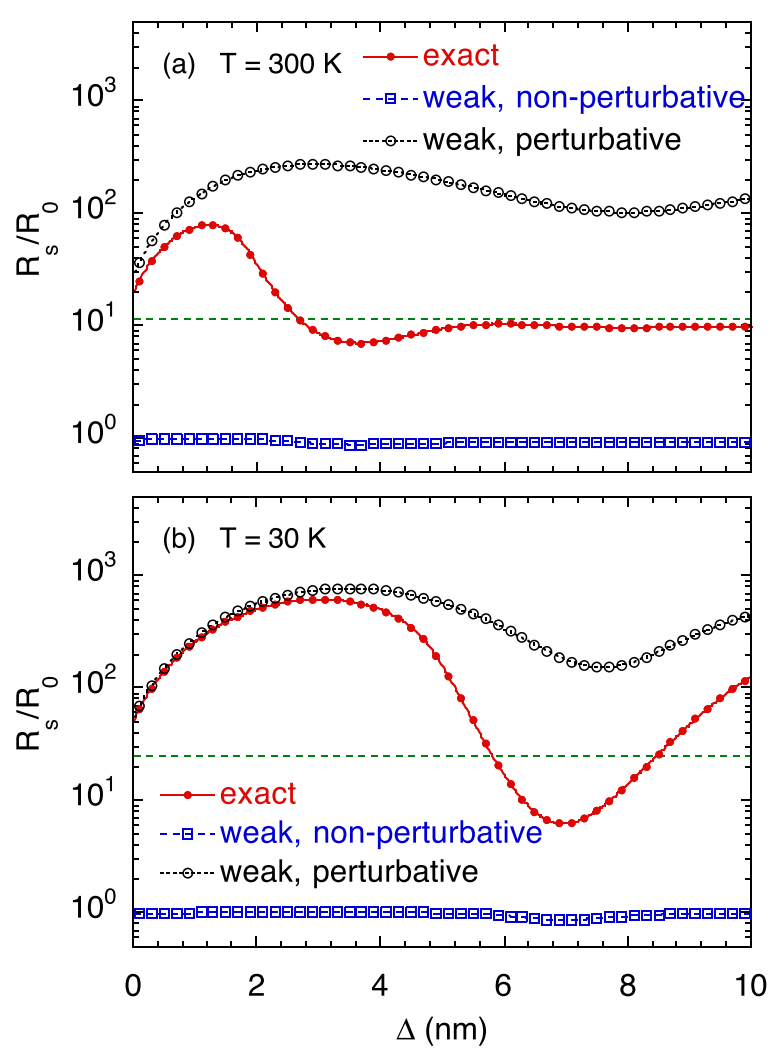

FIG. 6. Impurity-limited resistance $R_{s}$ of two impurities located on the wire axis at (a) $T=300$ and (b) $30 \mathrm{~K}$ as a function of the impurity separation $\Delta$ along the wire axis. The scattering potential energy is $v_{c}=183 \mathrm{meV} . R_{s}$ from the exact formula (red solid lines) and two approximations, $R_{s, \text { weak }}$ (blue dashed lines) and $R_{s, \text { weak }}^{B}$ (black dotted lines), are plotted. The horizontal dashed lines (green) show $2 R_{s}^{\text {single }}$; twice of the single-impurity resistance at $r_{0}=0$.

from the reservoirs. That is, $R_{s}$ is averaged by the incoming electrons with different kinetic energies (wavelengths). At $T=30 \mathrm{~K}$, the energy spectrum of the in-coming electrons is limited to be a very narrow range around the Fermi energy of the reservoirs and the phase interference lasts much longer distances. The characteristic length $\Delta_{\text {damp }}$, over which the phase interference is preserved, is roughly estimated by the broadness of the energy spectrum of the incident electrons, which is the temperature of the source and drain. Hence, $\Delta_{\text {damp }}$ is estimated by

$$
\Delta_{\text {damp }} \sim \frac{1}{\Delta k} \sim \frac{\hbar}{\sqrt{m k_{B} T}} .
$$

We find $\Delta_{\text {damp }} \approx 3 \mathrm{~nm}$ for $T=300 \mathrm{~K}$ and $\Delta_{\text {damp }} \approx 10 \mathrm{~nm}$ for $T=30 \mathrm{~K}$.

The extension to the case of three or more impurities is in principle straightforward, although numerical calculation becomes much more extensive and complicated. The essential features are, however, expected to be the same: The constructive interference among the impurities dominates as the axial separation between impurities is smaller than the characteristic length of the broadness of the energy spectrum of the incoming electrons from the reservoirs. As the impurity separation becomes larger, owing to the phase randomization caused by the broadness of the energy spectrum of incident electrons, the impurity-limited resistance becomes close to the value expected by the series resistance of singleimpurity.

\section{Space-average resistance of two correlated impurities}

When two impurities are located on the wire axis, the impurity-limited resistance is greatly exaggerated since the impurities become most resistive on the wire axis. In reality, however, impurities are doped at random in nanowires, and spatial position control of impurities is almost impossible. Therefore, the impurity-limited resistance averaged over the channel region is more realistic. This is also equivalent to the (non-averaged) resistance of long-channel nanowires where the impurity configuration is self-averaged.

Assuming that impurities are uniformly distributed in the nanowire and generating the positions, $\mathbf{R}_{01}$ and $\mathbf{R}_{02}$, of two impurities at random, the impurity-limited resistance $R_{s}$ $\left(\mathbf{r}_{01}, \mathbf{r}_{02}, \Delta\right)$ for each impurity configuration is calculated. The results are shown as a function of the impurity separation $\Delta$ in Fig. 7, where the resistances $R_{s}\left(\mathbf{r}_{01}, \mathbf{r}_{02}, \Delta\right)$ of 2000 different configurations of the two-impurity are shown. For comparison, similar plots obtained from the more elaborate tight-binding NEGF simulations for donor and acceptor impurities are shown in Fig. 8, in which the resistances of 500 different configurations of two impurities are plotted. ${ }^{50}$ The shape of the cross-section is square with the side length of $3.5 \mathrm{~nm}$, rather than sphere. However, the cross-sectional area is nearly the same as that of the circular nanowire employed in this study, and thus, the difference in shape is insignificant. Since many simulations under different impurity configurations are required to be performed, the scattering potential of ionized impurities is modeled by the analytical screened Coulomb potential along with the image charges to take into account the dielectric mismatch between the substrate and the gate-oxide. The impurity density in the substrate is assumed to be $10^{19} \mathrm{~cm}^{-3}$, and the screening length is set at $\lambda_{s c}=1.3 \mathrm{~nm}$. The transmission coefficient is calculated from the Fisher-Lee formula with the retarded

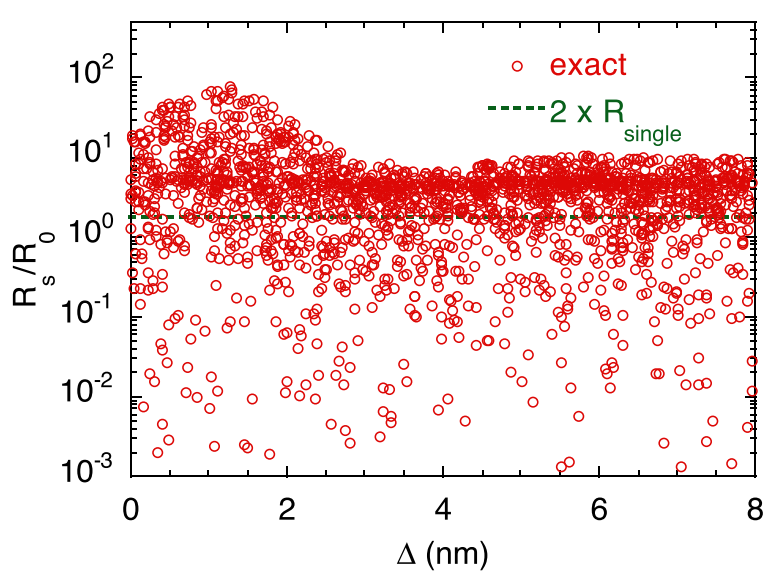

FIG. 7. Impurity-limited resistance $R_{s}\left(\mathbf{r}_{01}, \mathbf{r}_{02}, \Delta\right)$ of two correlated impurities as a function of the impurity separation $\Delta$ along the wire axis. The scattering potential energy is $v_{c}=183 \mathrm{meV}$ and the location of two impurities are generated at random and 2000 different configurations are shown. The horizontal dashed line (green) shows the value of the series resistance of two single-impurities, $2 \bar{R}_{\text {single }}$. 


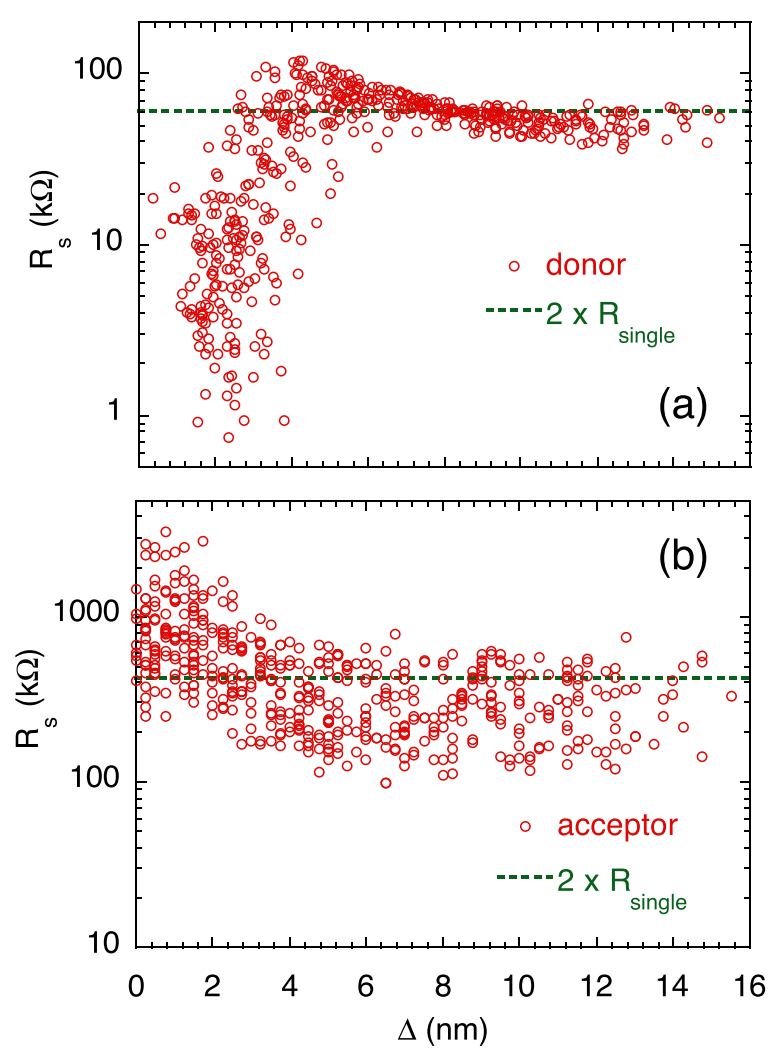

FIG. 8. Impurity-limited resistance $R_{s}$ obtained from the tight-binding NEGF simulations for the case of (a) donor impurities and (b) acceptor impurities in the square nanowire of the side length of $3.5 \mathrm{~nm}$. The scattering potential of ionized impurity is described by the screened Coulomb potential and the resistances for 500 different configurations of two impurities are shown.

Green function. ${ }^{51}$ The horizontal (green) dashed lines show $2 \bar{R}_{\text {single }}, \bar{R}_{\text {single }}$ being the space-average resistance of the single-impurity determined from the "single-impurity" NEGF simulations. There are some distinct features in between the present results and those from the NEGF simulations, for example, the dependence of the type of ionized impurities, etc. They mainly result from the long-range part of the screened Coulomb potential of impurities in the NEGF calculations. Nevertheless, an essential feature is common in these results: The constructive interference dominates for small $\Delta$ in $R_{s}$, whereas $R_{s}$ becomes close to the series resistances of two impurities, $2 \bar{R}_{\text {single }}$, with $\bar{R}_{\text {single }}$ being the space-average resistance due to single-impurity.

The space-average resistance $\bar{R}_{s}$ is calculated with the probability density of impurity position $p_{i m p}\left(\mathbf{R}_{0 r}\right)(r=1,2)$ by

$$
\begin{aligned}
\bar{R}_{s} & =\int d^{3} R_{01} d^{3} R_{02} p_{i m p}\left(\mathbf{R}_{01}\right) p_{i m p}\left(\mathbf{R}_{02}\right) R_{s}\left(\mathbf{r}_{01}, \mathbf{r}_{02}, \Delta\right) \\
& =\frac{1}{L} \int d \Delta \frac{1}{\left(\pi r_{s}^{2}\right)^{2}} \int d^{2} r_{01} d^{2} r_{02} R_{s}\left(\mathbf{r}_{01}, \mathbf{r}_{02}, \Delta\right),
\end{aligned}
$$

where $L$ is the channel length for the two-impurity system. Figure 9 shows the space-average resistance $\bar{R}_{s}$ of two correlated impurities at $T=300$ and $30 \mathrm{~K}$ as a function of the scattering potential energy $v_{c}$. The resistances from the exact formula $R_{s}$ and two approximations, $R_{s, \text { weak }}$ and $R_{s, \text { weak }}^{B}$, are
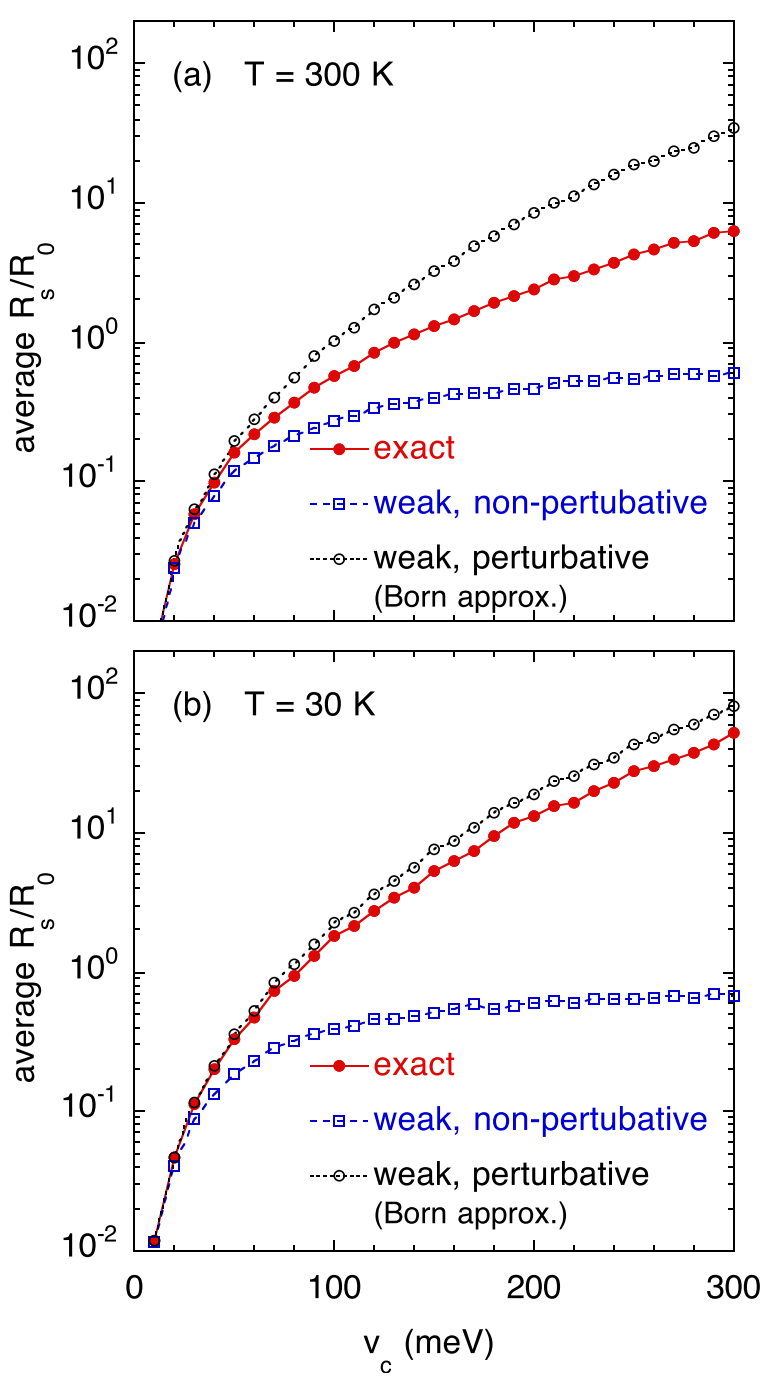

FIG. 9. Space-average impurity-limited resistance $\bar{R}_{s}$ of two correlated impurities as a function of the scattering potential energy $v_{c}$ for (a) $T=300 \mathrm{~K}$ and (b) $30 \mathrm{~K}$. The average resistances from the exact formula $R_{s}$ (red solid lines) and two approximations, $R_{s, \text { weak }}$ (blue dashed lines) and $R_{s, \text { weak }}^{B}$ (black dotted lines), are shown.

shown. It is clear that the two approximations greatly deviate from the exact results and, thus, the multiple-impurity cannot be properly described by the weak-coupling approximations unless the coupling strength is extremely small. In other words, both the full details of the transition matrix and the inclusion of the denominator $\left\langle T_{A}\right\rangle$ in $R_{S}$ are crucial even for space-average resistances. We should stress again that this is true as far as the scattering potentials due to multiple impurities are treated as a single-scattering potential so that the phase coherence among the impurities is fully preserved. Therefore, the perturbative Born approximation of the impurity scattering under the framework of the NEGF would greatly overestimate the impurity-limited resistance at room temperature. It is necessary to treat scattering due to multiple impurities nonperturbatively. As temperature is lowered, however, the average resistance $\bar{R}_{s, \text { weak }}$ under the Born approximation becomes much better.

Figure 10 shows the space-average resistance $\bar{R}_{s}$ of the two-impurity systems and $2 \bar{R}_{\text {single }}$ of the single-impurity systems at $T=300$ and $30 \mathrm{~K}$ as a function of the scattering 


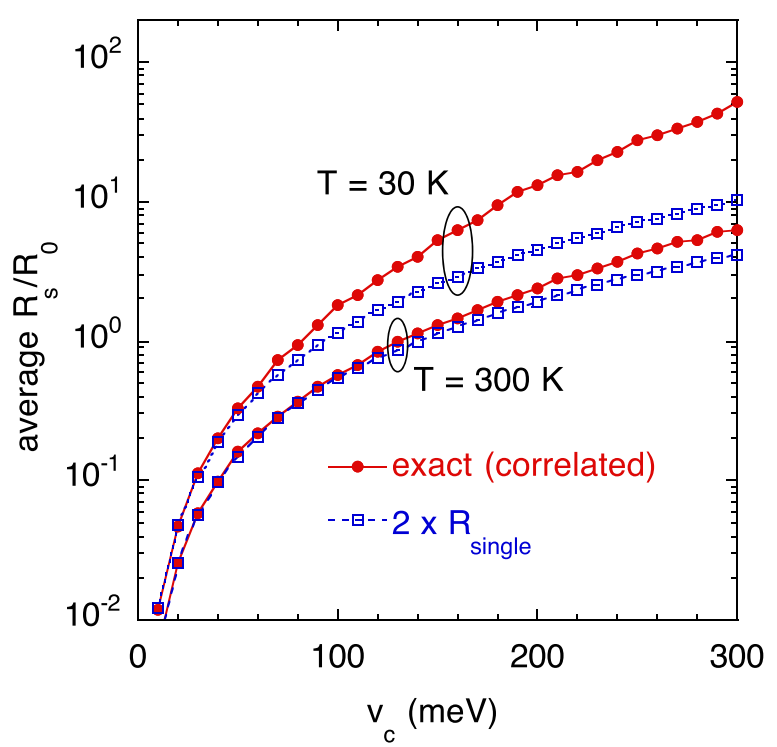

FIG. 10. Space-average resistance $\bar{R}_{s}$ of the two-impurity systems and $2 \bar{R}_{\text {single }}$ at $T=300$ and $30 \mathrm{~K}$ as a function of the scattering potential energy $v_{c} . \bar{R}_{\text {single }}$ is the space-average resistance of the single-impurity systems.

potential energy $v_{c}$. The two results at $T=300 \mathrm{~K}$ agree quite well up to large $v_{c}$ where the weak-scattering limit breaks down. Since $2 \bar{R}_{\text {single }}$ represents the uncorrelated series resistance of two impurities, this agreement implies that it is the phase randomization which cannot be taken into account properly in the weak-scattering approximations, leading to the deviations from the exact result as seen in Fig. 9. Therefore, each impurity under the uniform impurity distribution could be regarded as an independent scattering center, and phase interference among the impurities almost diminishes at room temperature. Notice again that the system is purely coherent and no energy dissipating scattering is included. This is exactly equivalent to the mechanism of "self-averaging," and thus, it is conjectured that the selfaveraging is caused by the phase randomization by the incoming electrons with broad energy spectrum from the reservoirs.

As the temperature is lowered, the phase randomization is not strong enough due to narrow energy spectrum of incoming electrons, and the phase interference is preserved even in the space-average resistance. Then, it is essential to treat the whole potential modulation induced by multiple impurities as a single scattering potential and to take into account the phase coherence among the impurities. As a result, the "single-impurity scattering" picture completely breaks down. It is also clear from Fig. 10 that as $v_{c}$ gets larger, the deviation between the two results become noticeable even at $T=300 \mathrm{~K}$. However, $v_{c}$ is unrealistically high there, and thus, in many cases, the space-average impuritylimited resistance at room temperature, which is equivalent to the ensemble average of the resistance in short-channel nanowires or the resistance in a long-channel nanowire, would be well described by the "single-impurity scattering" picture. In other words, the classical Ohm's law holds true as far as the average impurity-limited resistance is concerned.

Here, we would like to comment on the impurity (or electron) density dependence of the average resistance because of its crucial importance in technological applications. Under the present formulation, the impurity density dependence of the resistance is not so obvious as in the classical cases: The impurity density dependence is hidden in the form of the scattering potential operator (see Eq. (37)) and does not appear explicitly. On the other hand, the resistance is inversely proportional to the electron density, ${ }^{52}$ and this dependence explicitly appears in the classical expression of resistance. Of course, the impurity density dependence of the average resistance could be obtained from the present theory if one carries out similar calculations for more than three correlated impurities. In principle, this is possible, but analytical expressions of the reflection coefficients become extremely complicated as the number of impurity increases and actual numerical calculations are rather difficult, as mentioned in Sec. III B 2. However, it is expected that the "single-impurity scattering" picture, under which there is no correlation among impurities, holds true for multiple impurities at room temperature. In this case, the average resistance becomes trivially proportional to the inverse of the electron density. Therefore, it is conjectured that the impurity density dependence of the average resistance would be very similar to the classical one even if the phase coherence is fully taken into account by following the present formalism.

\section{CONCLUSIONS}

We have systematically investigated the impuritylimited resistance and the phase interference effects due to localized impurities in quasi-1D nanowires under the framework of the scattering theory.

Theoretical expressions of the impurity-limited resistance under the linear response regime have been derived from the Landauer formula in terms of the transmission and reflection probabilities obtained from the LippmannSchwinger equation. We have shown that the impuritylimited resistance under the weak-scattering limit coincides with that derived from the Boltzmann transport equation under the relaxation approximation unless the energy spectrum of the in-coming electrons from the reservoirs is very broad. From this finding, we have pointed out that the distinction of the impurity-limited resistances derived from the Landauer formula and that from the BTE could be made clear experimentally by varying the broadness of the energy spectrum of the electrons injected into the channel region. The deriving formulas have been applied to the cases where multiple localized impurities with the short-range scattering potential are doped in the nanowire and the exact theoretical expressions of the impurity-limited resistance have been derived.

We have shown explicitly through the numerical analyses that the scattering is so strong under the nanowire structures that the weak-scattering limit breaks down in most cases. When the impurity separation along the wire axis direction is comparable to or smaller than the characteristic length of the energy spectrum of the incoming electrons from the reservoirs, the constructive phase interference dominates and the resistance is much greater than the average 
resistance. As the separation becomes larger, however, it approaches the series resistance of the single-impurity resistance due to the thermal average taken over the incoming electrons from the reservoirs. These results are consistent with those from more elaborate tight-binding NEGF simulations in which the realistic screened Coulomb potential is employed for the donor and acceptor impurities. Under the uniform distribution of impurities, the space-average resistance of multiple impurities cannot be accurately described by the weak-scattering approximation, as far as the whole potential modulation due to impurities is treated as a single scattering potential. At room temperature, the spaceaveraged resistance of multiple impurities is very close to the value of the series resistance of the single-impurity resistance, and thus, each impurity could be regarded as an independent scattering center. This phase randomization is caused by the incoming electrons from the reservoirs with broad energy spectrum, and this is the physical origin of "self-averaging" under the fully coherent circumstances. As the temperature is lowered, the phase randomization is not strong enough due to the narrow energy spectrum of incoming electrons and the phase interference is preserved even in the space-average resistance. Then, it becomes crucial to treat the whole potential modulation induced by multiple impurities as a single scattering potential and to fully take into account the phase coherence among the impurities.

Finally, we would like to comment on the form of the scattering potential employed in the present study. In reality, the scattering potential always contains the long-range potential component and the detail shape of the long-range part of the potential indeed becomes crucial to predict the transport properties of nanowires quantitatively. In particular, it has been reported ${ }^{24}$ that the impurity-limited resistance greatly changes, depending on the sign of the charge of ionized impurities; acceptor impurities are more resistive than donor impurities. This is, however, trivially explained by the fact that the long-range part of the Coulomb potential of the acceptor impurity blocks electron wave propagation, whereas the donor impurities yield the potential depressions which are less resistive for free carriers. In fact, the phase interference among the ionized impurities is somewhat smoothed and less violent under the realistic scattering potential because of the long-range nature of the potential, as shown in Figs. 7 and 8. Furthermore, since the dominant role in scattering-limited resistance is played by the short-range part of the scattering potential, it is expected that the present results would well represent the essential features of the impurity-limited resistance in the nanowire even under more realistic impurity scattering potentials.

\section{ACKNOWLEDGMENTS}

We would like to acknowledge the financial support from Ministry of Education, Science, Sports, and Culture under Grant-in-Aid for Scientific Research (B) (No. 15H03983).

${ }^{1}$ J. Appenzeller, J. Knoch, M. Bjork, H. Riel, H. Schmid, and W. Riess, IEEE Trans. Electron Devices 55, 2827 (2008).
${ }^{2}$ V. Schmidt, J. V. Wittemann, S. Senz, and U. Gösele, Adv. Mater. 21, 2681 (2009).

${ }^{3}$ R. Rurali, Rev. Mod. Phys. 82, 427 (2010).

${ }^{4}$ S. Rustagi, N. Singh, Y. Lim, G. Zhang, S. Wang, G. Lo, N. Balasubramanian, and D. Kwong, IEEE Electron Device Lett. 28, 909 (2007).

${ }^{5}$ J. Martinez, R. V. Martinez, and R. Garcia, Nano Lett. 8, 3636 (2008).

${ }^{6}$ M. Lee, Y. Jeon, T. Moon, and S. Kim, ACS Nano 5, 2629 (2011).

${ }^{7}$ J.-P. Colinge, Solid-State Electron. 48, 897 (2004).

${ }^{8}$ W. Lu and C. M. Lieber, J. Phys. D: Appl. Phys. 39, R387 (2006).

${ }^{9}$ N. Sano, K. Matsuzawa, M. Mukai, and N. Nakayama, IEEE Int. Electron Devices Meet., Tech. Dig. 275-278 (2000).

${ }^{10}$ N. Sano, K. Matsuzawa, M. Mukai, and N. Nakayama, Microelectron. Reliab. 42, 189 (2002).

${ }^{11}$ J.-S. Yoon, T. Rim, J. Kim, K. Kim, C.-K. Baek, and Y.-H. Jeong, Appl. Phys. Lett. 106, 103507 (2015).

${ }^{12}$ W. Kohn and J. M. Luttinger, Phys. Rev. 108, 590 (1957).

${ }^{13}$ J. R. Barker, Semicond. Sci. Technol. 19, S56 (2004).

${ }^{14}$ M. Bohr, IEEE Trans. Nanotechnol. 1, 56 (2002).

${ }^{15}$ N. Sano, A. Hiroki, and K. Matsuzawa, IEEE Trans. Nanotechnol. 1, 63 (2002).

${ }^{16}$ N. Sano, Appl. Phys. Lett. 85, 4208 (2004).

${ }^{17}$ N. Sano, Phys. Rev. Lett. 93, 246803 (2004).

${ }^{18}$ M. V. Fischetti, S. Jin, T.-W. Tang, P. Asbeck, Y. Taur, S. E. Laux, M. Rodwell, and N. Sano, J. Comput. Electron. 8, 60 (2009).

${ }^{19}$ T. Markussen, R. Rurali, M. Brandbyge, and A.-P. Jauho, Phys. Rev. B 74, 245313 (2006).

${ }^{20}$ T. Markussen, R. Rurali, A.-P. Jauho, and M. Brandbyge, Phys. Rev. Lett. 99, 076803 (2007).

${ }^{21}$ R. Rurali, T. Markussen, J. Sune, M. Brandbyge, and A.-P. Jauho, Nano Lett. 8, 2825 (2008).

${ }^{22}$ F. Iori, S. Ossicini, and R. Rurali, J. Appl. Phys. 116, 074303 (2014).

${ }^{23}$ D. Sharma and G. Fagas, J. Phys. Chem. C 119, 11934 (2015).

${ }^{24}$ M. P. Persson, H. Mera, Y.-M. Niquet, C. Delerue, and M. Diarra, Phys. Rev. B 82, 115318 (2010).

${ }^{25}$ A. Martinez, M. Aldegunde, N. Seoane, A. Brown, J. Barker, and A. Asenov, IEEE Trans. Electron Devices 58, 2209 (2011).

${ }^{26}$ Y.-M. Niquet, H. Mera, and C. Delerue, Appl. Phys. Lett. 100, 153119 (2012).

${ }^{27}$ D. Jena and A. Konar, Phys. Rev. Lett. 98, 136805 (2007).

${ }^{28}$ S. Jin, M. V. Fischetti, and T.-W. Tang, J. Appl. Phys. 102, 083715 (2007).

${ }^{29}$ M. T. Björk, H. Schmid, J. Knoch, H. Riel, and W. Riess, Nat. Nanotechnol. 4, 103 (2009).

${ }^{30} \mathrm{M}$. Aldegunde, A. Martinez, and J. Barker, IEEE Trans. Electron Devices 33, 194 (2012).

${ }^{31}$ S. Sylvia, K. Habib, M. Khayer, K. Alam, M. Neupane, and R. Lake, IEEE Trans. Electron Devices 61, 2208 (2014).

${ }^{32}$ In addition, the difference of the local density of states between the lead and the channel also contributes to the channel resistance. We ignore this contribution by assuming that the nanowire has the same shape through the leads and the channel.

${ }^{33} \mathrm{We}$ ignore the short-range electron-electron interaction in the present study and this part is left for future study.

${ }^{34}$ M. DiVentra, Electrical Transport in Nanoscale Systems (Cambridge University Press, UK, 2008).

${ }^{35}$ J. R. Taylor, Scattering Theory: The Quantum Theory of Nonrelativistic Collisions (Dover, New York, 2000), p. 133.

${ }^{36}$ R. G. Newton, Scattering Theory of Waves and Particles (Dover, New York, 2002), p. 228.

${ }^{37}$ C. Jacoboni and L. Reggiani, Rev. Mod. Phys. 55, 645 (1983).

${ }^{38} \mathrm{C}$. Jacoboni, Theory of Electron Transport in Semiconductors: A Pathway from Elementary Physics to Nonequilibrium Green Functions (Springer, Berlin, Heidelberg, 2010).

${ }^{39}$ N. Ashcroft and N. Mermin, Solid State Physics (Saunders College, Philadelphia, 1976).

${ }^{40}$ D. Chattopadhyay and H. J. Queisser, Rev. Mod. Phys. 53, 745 (1981).

${ }^{41}$ In addition, $\mu_{L}$ is assumed to be well above the lowest subband energy $\varepsilon_{A}$. Notice that $\mu_{L}$ has in general nothing to do with the carrier density of the channel region.

${ }^{42}$ M. Moško and P. Vagner, Phys. Rev. B 59, R10445 (1999).

${ }^{43}$ This is why the transport equation for the density matrix or the lesser Green function are required to be coupled.

${ }^{44}$ J. Lee and H. N. Spector, J. Appl. Phys. 54, 3921 (1983). 
${ }^{45}$ J. Lee and H. N. Spector, J. Appl. Phys. 57, 366 (1985).

${ }^{46}$ The extension to space-dependent $v_{c}$ allows one to study the effects of the long-range part of the scattering potential.

${ }^{47}$ J. Salfi, I. Savelyev, M. Blumin, S. Nair, and H. Ruda, Nat. Nanotechnol. 5, 737 (2010)

${ }^{48}$ Under the nanowire structure, the Coulomb potential is screened by the gate-oxide and high-doped gate, and thus, the present estimate of the scattering parameter is somewhat overestimated.
${ }^{49}$ Strictly speaking, the weak-scattering limit is also necessary for the claim to be true.

${ }^{50}$ N. Sano, M. R. Zulhidza, Y. Kaneno, S. Honda, A. Ueda, and K. Yoshida, J. Phys.: Conf. Ser. 647, 012028 (2015).

${ }^{51}$ D. S. Fisher and P. A. Lee, Phys. Rev. B 23, 6851 (1981).

${ }^{52}$ The carrier density dependence in resistance is also implicitly included in the relaxation time through the scattering processes in the classical cases. 